\title{
Thermodynamic Characterization of High-Speed and High-Enthalpy Plasma Flows
}

\author{
Y. Babou ${ }^{*}, 1$, D. Lequang ${ }^{1,2}$, O. Chazot ${ }^{1}$, S. T. Surzhikov ${ }^{3}$, A.S. Dikaljuk ${ }^{3}$, A. Panarese ${ }^{4}$, G. Cicala ${ }^{5}$, \\ S. Longo ${ }^{4,5}$, J. Hoffman ${ }^{6}$, Z. Szymanski ${ }^{6}$, A. Kaminska ${ }^{7}$, M. Dudeck ${ }^{8}$ and D. Vacher ${ }^{2}$ \\ ${ }^{1}$ Aeronautics and Aerospace Department, von Kármán Institute for Fluid Dynamics, Chaussée de Waterloo 72, 1640 \\ Rhode-Saint-Genèse, Belgium \\ ${ }^{2}$ Clermont Université, Université d'Auvergne, LAEPT, BP 80026, F-63000, Clermont-Ferrand, France \\ ${ }^{3}$ Institute for Problems in Mechanics Russian Academy of Sciences, 101-1, Vernadskogo prosp. Moscow, 119526, Russia \\ ${ }^{4}$ Department of Chemistry, University of Bari, via Orabona 4, 70126 Bari, Italy \\ ${ }^{5}$ CNR-IMIP, Bari Section, via Amendola 122/D, 70126 Bari, Italy \\ ${ }^{6}$ Institute of Fundamental Technological Research, Polish Academy of Science, Adolfa Pawinskiego 5, 02-106 Warsaw, \\ Poland \\ ${ }^{7}$ Institute of Electric Power Engineering, Poznan University of Technology, ul. Piotrowo 3A, 60-965 Poznan, Poland \\ ${ }^{8}$ Institut dAlembert, Universit Pierre et Marie Curie (Paris 6), 4 place Jussieu, 75252 Paris, Cedex, France
}

\begin{abstract}
This contribution proposes a description of selected experimental activities conducted in aerospace sciences and dedicated to generate experimental data to assess atmospheric entry plasma models. In order to provide comprehensive set of experimental data, high enthalpy shock tube facilities have been developed to generate plasma representative of entry plasma for broad range of trajectory entry conditions. The shock-heated plasma is obtained through adiabatic compression and the resulting post-shock plasma flow exhibits thermodynamic state analogous to actual entry plasma. However, significant insight can be obtained through experiments conducted also with non-equilibrium plasma flows obtained with other methods. The typical methodologies adopted to provide experimental data of interest to enhance entry plasma modeling are sketched for four distinct non-equilibrium plasma kinds produced respectively by four specific ground facilities. The contribution firstly will consider experimental campaigns conducted with a high enthalpy shock tube in order to document in absolute radiance the radiative signature in the UV spectral range of an Earth entry plasma. Then, the investigations of the interaction between a shock wave and an electrical discharge will be described. These investigations were performed to identify the role of the internal degrees of freedom of molecular gases on the propagation of the shock. Also, the contribution covers investigations devoted to the thermodynamic state characterizations by means of spectroscopic diagnostics in the cases of the non-equilibrium plasmas flows generated by plasma wind tunnels. The examination of the Saha-Boltzmann equilibrium is proposed in the case of a subsonic plasma flow. And at last, the characterization methods of air supersonic plasma jet are presented and the 2D distributions of the subsequently measured plasma properties are documented for a straight comparisons with non-equilibrium plasma jet computations.
\end{abstract}

Keywords: Abel transform, multi-temperature model optical emission spectroscopy, non-equilibrium plasma, particle in cell Monte Carlo computation, plasma wind tunnel, saha balance, shock tube, supersonic plasma.

\section{INTRODUCTION}

During a hypersonic reentry into a planetary atmosphere, large amount of the free stream kinetic energy is converted, across a strong bow shock through inelastic collisions, into internal energy of the gas. After the shock, in the relaxing flow, competition between coupled collisional and radiative

*Address correspondence to this author at the Aeronautics and Aerospace Department, von Kármán Institute for Fluid Dynamics, Chaussée de Waterloo 72, 1640 Rhode-Saint-Genèse, Belgium; Tel: + 322359 96 37; Fax: + 32235996 00; E-mail: yacine.babou@vki.ac.be processes occurring at different time scales rules the energy distribution in the dissociating, ionizing and radiating flow. As a consequence, the post shock plasma, designated as entry plasma, is typically characterized by energy levels no more ruled by the Maxwell-Boltzmann equilibrium distribution. The accurate prediction of non-equilibrium distributions is a decisive task for reliable prediction of the radiative heating, determining the design optimization of the space vehicle heat shield. The validation of non-equilibrium models developed for entry plasmas should be conducted via measurements performed during real-flight experiments [1]. Due to the elevated cost of these experiments and the 
difficulty to obtain low uncertainty measurements in such harsh environment, this information is far from being complete today. Therefore, the continuous improvement of advanced chemico-physical and plasma models for entry plasmas is performed on the basis of experiments carried out on ground with dedicated facilities operated with well-suited diagnostics to examine in details non-equilibrium processes. In practice, quantitative characterization of excited states populations, gas temperature and electron densities is made via the standard Optical Emission Spectroscopy (OES) diagnostic due to its moderate cost and easy implementation, conversely to a laser-based optical diagnostic. The present contribution proposes an outline of current research activities conducted in the aerospace sciences by focusing on up-todate experimental investigations carried out on four specific ground facilities. The objective is to depict the common methodologies employed to produce experimental data of interest to assess thermodynamic state of the investigated plasma. The investigations encompass the characterization of four distinct non-equilibrium plasma kinds with the aim to produce new data which can be directly used to assess the performances of non-equilibrium plasma flow computations.

High enthalpy shock tube experiments aim to investigate the kinetic processes taking place after a pressure discontinuity wave traveling through a gaseous mixture representative of planetary atmosphere conditions (composition and pressure). Such shock wave (SW) is generated by rupturing a diaphragm between high and low pressure regions. To achieve a shock wave representative of the entry plasma requires to generate high energy pulse to dissipate a large amount of energy over hundreds of microseconds to adiabatically compress the gas. Experiments dedicated to the measurements of radiation emitted by the post-shock plasma have started in the second half of twentieth century, triggered by the requirements for space exploration missions. The first attempts of recording radiation in absolute units behind the front of shock waves generated with a shock tube operating with air and $\mathrm{CO}_{2}-\mathrm{N}_{2}$ mixtures have been reported in [2] and [3] respectively. However the instrumentation available at the time could not provide desirable quality of measurements. Since the last decade, development of high-speed and high-resolution CCD cameras and technology of digital registration of optical signals revitalized high enthalpy shock tube experimental activities dedicated to Earth, Mars, Venus and Titan atmospheric entry (e.g. [4-10]). The fruitful harvest of experimental spectra has been conducive to assess performances of standard chemical models adopted in aerospace engineering, such as the so-called twotemperatures Park model $[11,12]$ or detailed kinetic models developed by plasma scientists such as electronic or vibrational specific Collisional-Radiative (CR) models. For instance, the Tube a Choc Marseille 2 (TCM2) facility which is middle-sized free piston shock tunnel [13] has been employed to document the $\mathrm{CN}$ and $\mathrm{C}_{2}$ absolute intensity behind a strong shock wave in a $\mathrm{CO}-\mathrm{N}_{2}$ mixture for various operating conditions. Significant discrepancies between recorded spectral intensities and those rebuilt with species densities determined with the Park model have pointed out the needs for modeling improvement to reproduce correctly $\mathrm{CN}$ and $\mathrm{C}_{2}$ emission profiles [14]. Also, the Electric Arc Shock Tube (EAST) facility at NASA Ames, which operates with an electric arc-heated driver, was recently used to procure spectral intensities emitted by the plasma behind a shock representative of Earth entry [4]. Very good agreement has been obtained between the measured post-shock emission profiles of $\mathrm{N}$ and $\mathrm{O}$ electronic transitions and those rebuilt with up-to-date electronic specific $\mathrm{CR}$ model for $\mathrm{N}$ and $\mathrm{O}$ species [15], meanwhile pointing out the inadequacy of standard two-temperatures models. Currently, the experimental research efforts are oriented to record intensities in UV and more particularly VUV spectral ranges, both exhibiting moderate and strong self-absorption pending on the contributive radiative systems. Measurement in the VUV spectral domain requires in particular specific arrangement to guarantee optical path under well controlled vacuum. The recent EAST shock tube experiments with carbonaceous mixture, focusing on VUV measurements, provided up-to-date experimental data to validate the $\mathrm{CO}$ radiative properties [16]. A typical high enthalpy shock tube experimental campaign conducted at Institute for Problems in Mechanics (Russian Federation) to document the UV radiative signature of a post-shock plasma representative of Earth entry is described in Part II.

In addition to high enthalpy shock tube experiments, significant insight can be obtained through experiments conducted with non-equilibrium plasma flows not necessarily representative of the entry plasma. Indeed, there is a broad range of non-equilibrium plasma situations that can be considered, in order to examine independently some specific mechanisms or to identify limitations of adopted entry plasma descriptions. For example an interesting and still open question is the role of the internal degrees of freedom of molecular gases on the propagation of the shock. This problem can be tackled through a campaign of comparative experiments involving an atomic and a molecular plasma, e.g. Ar and $\mathrm{N}_{2}$ (respectively the most important atomic and molecular gas in aerospace studies) under the same experimental conditions [17]. Until now, such comparative measurements have only been performed at University of Bari. Several research groups have found and observed that the propagation velocity of SW in ionized gas is higher than that in nonionized gas and also the shock shapes differ in plasma with respect to neutral gas because they broaden, split and attenuate. Many researchers [18, 19] believed that the broadening, splitting and acceleration of shock in gas discharges are due to conventional thermal phenomena: Gas heating and temperature inhomogeneity. From a theoretical point of view Aital and Subramaniam [20] predicted that the splitting of the signal as well as the spread and attenuation of weak SW, as revealed by laser deflection measurements, are due to a temperature gradient along the axis and radius of the discharge tube, as well as to a wall shear that causes a marked curvature of the shock front (near-wall regions). Subsequently, the same authors [21] showed that the measurements of the laser deflection signal recovery dependent on the direction of discharge electric field are further evidence of plasma effects. The recovery distance measured in the afterglow when the SW travels from the anode to the cathode is indeed longer than that from the cathode to the anode in glow discharge because of the cathode (i.e. the hot electrode) proximity. For example Macheret et al. [19] obtained a direct proof of the thermal mechanism by pulsing the discharge and demonstrated 
experimentally and numerically that the changes in SW velocity and broadening are explained by a heating of the gas and a radial temperature gradient, respectively. However, several other authors argued that the transformations of SW (such as the splitting in two waves, the significant broadening of SW front and the average velocity increase) are due to various plasma-specific effects such as electric double layer, ion-acoustic waves and long range interaction [22-24]. Thus, for the above cited phenomena two kinds of interaction have been invoked and consequently two disparate explanations have been proposed. Whether or not the SW velocity is enhanced for a thermal $[18,19]$ or a non thermal (i.e. electrostatic interaction with the plasma) [22-24] mechanism; SW propagation in plasma is still a contentious issue being addressed in Part III.

In the field of aerospace science, in addition to shock tube experiments, diverse Plasma Wind Tunnel (PWT) facilities have been constructed for Heat Protective Material (HPM) testing purpose to support the development of Thermal Protection System (TPS) for space mission. PWTs are employed to provide a steady axisymmetric subsonic or supersonic plasma flow of large diameter $(\gtrsim 10 \mathrm{~cm})$ in order to test insulating performance of material samples, candidates for TPS applications, undergoing thermal heat fluxes representative of those encountered during the entry trajectory into planetary atmosphere ( 1-20 MW.m $\left.{ }^{-2}\right)$. Different methods based on electric discharge heating are used to generate high enthalpy plasmas. PWTs based on Direct-Current (DC) discharge are so-called arc-jets or DC-Plasmatron [27, 28]. PWTs using Inductively Coupled Plasma (ICP) torch, based on Radio-Frequency (RF) discharge, are so-called RF-Plasmatron [25, 26]. Both types are employed to test HPM under duplicated flight conditions, nevertheless it might be stressed that currently higher enthalpy range is covered with DC-Plasmatrons, making them well appropriate to test HPM undergoing heat fluxes beyond $\sim 15 \mathrm{~kW} \cdot \mathrm{m}^{-2}$. PWTs are still under exploited nowadays for the task of non-equilibrium plasma description assessment, despite their ability to offer a steady plasma flow jet with varied thermodynamic situations and their practical advantages in comparison with shock tube flows of shorter runtime. For example in subsonic plasma flows the thermodynamic equilibrium is rarely achieved since the elevated temperature of electrons produced in the $\mathrm{DC}$ or RF discharge region, plasma jet radiative losses, velocity and temperature gradients can result in significant departure from chemical and thermal equilibrium. Electron density determination on the basis of OES diagnostics in air subsonic plasma jet produced at low pressure with the VKI-Plasmatron facility were reported in [29]. Differences between electron density estimations at equilibrium and measurements evidenced for a non negligible departure from Saha-Boltzmann equilibrium similarly to plasma undergoing recombination. Subsonic plasma jets generated with ICP torch of more modest size than PWT facilities can be smartly exploited for the specific task of experimental assessment of Collisional-Radiative models. As reported in [30], OES characterisation of an atmospheric pressure nitrogen/argon plasma flowing at high velocity through a water-cooled testsection, to control its recombination time, have permitted to assess a vibrational specific $\mathrm{CR}$ model of $\mathrm{N}_{2}$ excited states. The Part IV of the contribution proposes a detailed examination of the thermodynamic state by means of OES of a low pressure subsonic plasma flow generated with nitrogen mixture by the DC-Plasmatron facility at Poznan University of Technology (Poland).

Plasma flows presenting considerable departure from equilibrium can be achieved with PWTs by using a convergent or convergent-divergent nozzle to generate a supersonic plasma jet. Many aspects of supersonic expanding plasma flows are of major interest for fundamental plasma physics and chemical-kinetics investigations. Because electrons and heavy species have important mass difference, a charge separation might occur, inducing then a self-consistent electromagnetic field that can influence transport of charged species. Also, a short flow residence time (due to its high velocity) is conducive for detailed investigations of the coupling between the ionization-recombination processes and the transport, and even for rebuilding rates for electronic kinetic processes [31]. In addition to the strictly expanding plasma, underexpanded supersonic plasma jets offer successive shocks structures. They typically exhibit various stages: In the supersonic expansion the plasma flow tends to be chemically frozen while in the post shock regions the plasma flow is subsonic. Comprehensive theoretical and numerical studies of the entire underexpanded jet are still scarce due to the wide range of flow regimes with more or less pronounced departure from equilibrium for the electronic, vibrational, rotational, and translational degrees of freedom (especially in shock regions). Besides the difficulties to predict supersonic plasma jet, inherent to the complexity of the flow, the reliability of standard OES methods used to rebuild plasma flow properties might be questioned. For instance OES investigations of a supersonic plasma jet generated with argon mixtures by an ICP torch have shown complete disagreement between electron temperature profiles obtained on the basis of two distinct rebuilding methods [32]. OES diagnostics applied to air underexpanded plasma jet generated by VKI-Plasmatron facility, available at von Karman institute (Belgium), are presented and discussed in Part V. The plasma parameters (electronic excitation temperature and electron density) were rebuilt adopting conventional approaches to characterized radially and axially the jet. The resulting $2 \mathrm{D}$ distributions of plasma parameters can be straightly employed to assess prediction of supersonic plasma flow coupled with detailed description of kinetic processes.

\section{HIGH ENTHALPY SHOCK TUBE PLASMA}

\section{Experiments}

The scheme of the shock tube facility developed at Institute of Mechanics of Moscow State University by P.V. Kozlov, Yu.V. Romanenko, O.P. Shatalov is presented in Fig. (1). Inner diameter of all 3 seamless tubes which are the sections of tube is $50 \mathrm{~mm}$. Use of seamless stainless steel tubes allowed to exclude jumps of temperature and density on the junctions of tube in the registration section. High Pressure Chamber (HPC) is filled with $0.5\left(\mathrm{H}_{2}+1 / 2 \mathrm{O}_{2}\right)+0.5 \mathrm{He}$ 


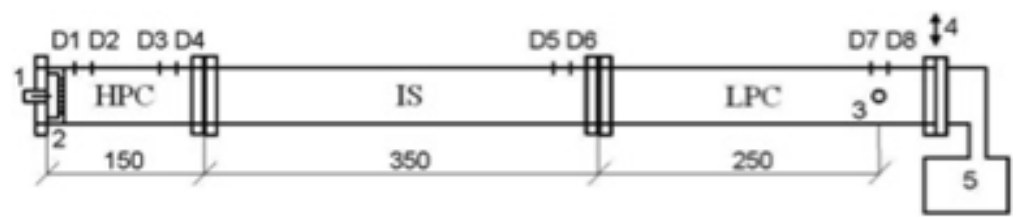

Fig. (1). The scheme of double-diaphragm shock tube. 1-spark-plug; 2-antechamber; 3-quartz window; 4-vent damper; 5-buffer tank. HPC -high-pressure chamber; IS - intermediate section; LPC - low-pressure chamber.

gas mixture and operated in detonation regime. Mixing of the mixture is performed in HPC. Hydrogen is added to preliminary prepared mixture of helium and oxygen. Stability of HPC operation in detonation regime substantially depends on the duration of mixing of explosive (combustible) mixture. Spark-plug is used at the rear end of the chamber for burning of this mixture. Spark-plug operates in the self-breakdown regime. Energy stored on the discharge high-voltage capacitors is less than $100 \mathrm{~mJ}$, duration of the discharge is $300-400$ ns. Capacitors and spark-plug are low-inductance circuit which provide nanosecond duration time of discharge. Antechamber is situated between blind flange with the spark-plug and HPC section. It promotes the rapid development of plane combustion front in HPC. Cassettes with copper diaphragms and cross-like cutters are situated between the sections of the tube. The width of the diaphragm between HPC and intermediate section (filled with helium) is $130 \mu \mathrm{m}$ and the width of diaphragm between intermediate section and lowpressure chamber (LPC) is $80 \mu \mathrm{m}$. Configuration of the double-diaphragm shock-tube described above when HPC filled with mixture $\mathrm{H}_{2}: \mathrm{O}_{2}: \mathrm{He}=2: 1: 3$ at pressure $438 \mathrm{kPa}$ and LPC is filled with air $\left(78 \%\right.$ of $\mathrm{N}_{2}$ and $21 \%$ of $\left.\mathrm{O}_{2}\right)$ at pressure $133 \mathrm{~Pa}$ allows to reach the shock velocity equal to $7 \mathrm{~km} / \mathrm{s}$. Reproducibility of gas parameters behind the shock wave is about $2 \%$ and in general depend on the quality of mixing of explosive mixture in HPC. Buffer tank and low pressure chamber are evacuated to the pressure $10^{-4} \mathrm{~Pa}$ using the dry pump system with leakage lower than $0.001 \mathrm{~Pa} / \mathrm{min}$. Pure gases of AGA company are used in order to produce the gas mixtures for the experiments.

Resulting spectral resolution, due to the ICCD camera and spectrograph combination, is about $0.33 \mathrm{~nm}$ for the diffraction grating of 150 groves per millimeter. The sensitivity region of ICCD camera is within the range of wavelengths $170-870 \mathrm{~nm}$. The measurements are performed within the wavelength region $200-850 \mathrm{~nm}$. Four overlapping sub-regions were chosen for the whole region $200-850 \mathrm{~nm}$. In order to eliminate influence of the spectra of radiation from second-order of grating on the measured spectra in the wavelength region $400-750 \mathrm{~nm}$ filters are installed in front of the spectrograph. Spectral radiation emitted by shock-heated gases is registered by ICCD-camera. From the opposite window the temporal dependence of integral signal through the quartz optical fiber is registered by means of photoelectric multiplier FEU-100. The signal from electric photomultiplier is registered using the digital oscilloscope. The spectral region of such scheme is $200-850 \mathrm{~nm}$ due to the transmissivity of optical fiber and the sensitivity region of photomultiplier. It is possible to install monochromator between the FEU-100 and optical fiber in order to register the temporal behavior of radiation in given regions of a spectrum. Temporal resolution of such scheme is $1 \mu$ s due to the geometry of optical scheme, can be improved up to $0.1 \mu \mathrm{s}$. The typical radiative signature recorded in UV spectral range for various operating conditions, presented in Fig. (2), point out the rise of emission with the shock velocity for a given working gas. An augmentation of 60 percent of the shock velocity leads to an increase of two order of magnitudes the $\mathrm{N}_{2}^{+}$peak intensity located at approximatively $380 \mathrm{~nm}$.

\section{SHOCKWAVE-ELECTRICAL DISCHARGE INTERACTION}

\section{Experiments}

The investigations described here below consider shock waves, generated by the Bari acoustic shock tube, propagating in weakly ionized discharge. The aim of such experiment is to compare and contrast the results of direct current discharge in atomic Ar and in molecular $\mathrm{N}_{2}$ and to uncover the role of thermal and non thermal effect in heat transfer. The characteristics of DC discharge, i.e. voltage, resistance and power of discharge, are obtained by electrical characterization of the plasma. The laser deflection technique is used to measure the structure and velocity of shock wave. The passage of shock wave creates a density gradient that permits to evaluate the propagation velocity of $\mathrm{SW}$ and to monitor the SW evolution. In Fig. (3a) the schematic of the experimental set-up is shown. The apparatus essentially consists of a Pyrex discharge tube, whose length and inner diameter are $8 \mathrm{~cm}$ and $4.3 \mathrm{~cm}$, respectively, and a spark gap which generate the shock wave. A DC supply ignites a non-equilibrium discharge in the discharge tube between a pair of stainless steel annular electrodes. The spark gap is made by a pair of stainless steel cylindrical electrodes separated by $7 \mathrm{~mm}$, as sketched in the circuit diagram of spark gap of Fig. (3b). The acoustic shock wave is produced by the spark gap driven by a triggered home-made spark switch, a high voltage capacitor $(0.5 \mu \mathrm{F})$, and a DC supply $(50 \mathrm{KV})$. The stored pulse energy is about $25 \mathrm{~J}$, as a voltage of $10 \mathrm{KV}$ is applied to the spark gap electrodes. The circuit diagram of DC discharge of Fig. (3c) shows that a ballast resistance, $\mathrm{R}_{B}=20 \mathrm{~K} \Omega$, is connected in series with plasma resistance, $R_{p l}$. This resistive ballast provides a positive resistance that limits the current through the tube and, therefore, prevents the DC supply to be destroyed or to fail. Besides $R_{B}$ makes the discharge stable at contemplated current range. In the circuit diagram of DC discharge is also shown the voltage divider that allows us to measure the discharge voltage. By varying the discharge current in the range $(0-90 \mathrm{~mA})$, it is possible to study the 


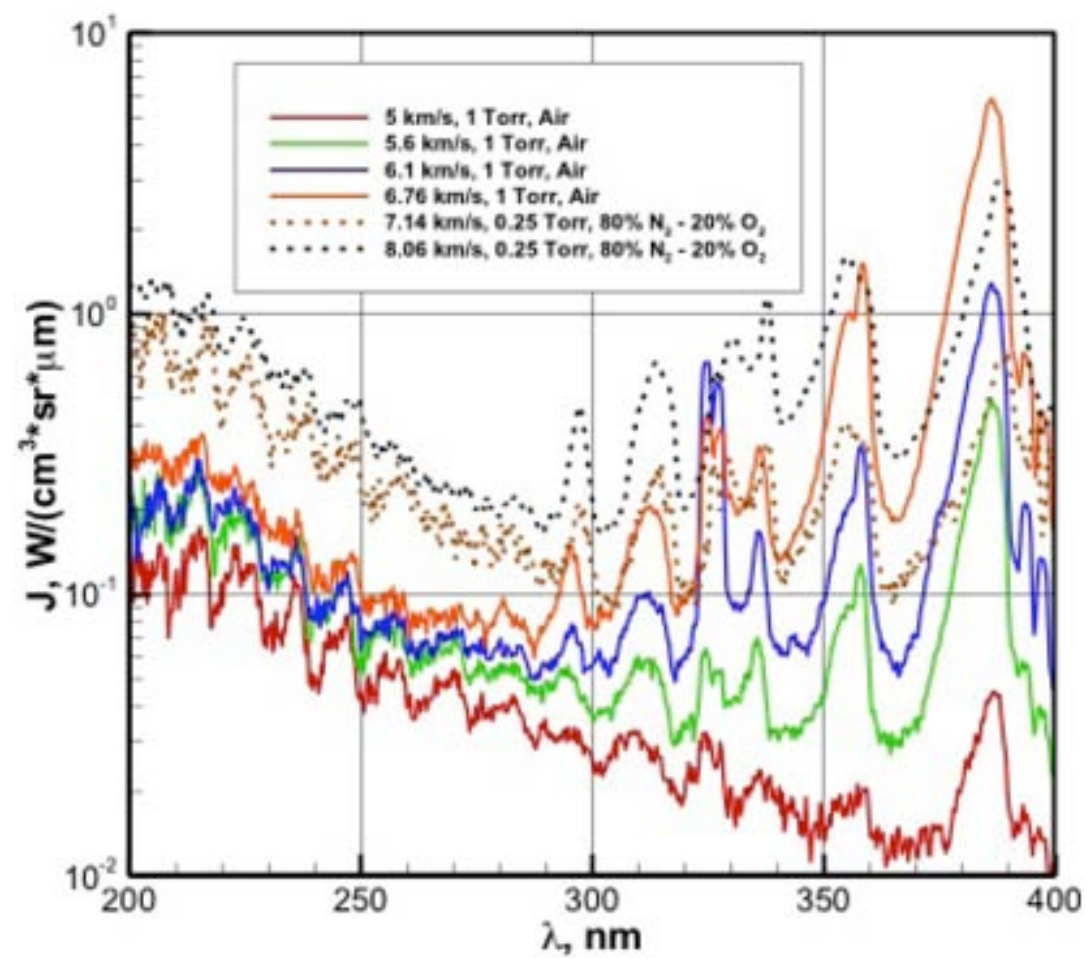

Fig. (2). Non-equilibrium spectral emissivity of the shock-heated ambient air - solid lines; $80 \% \mathrm{~N}_{2}-20 \% \mathrm{O}_{2}$ gas mixture - dashed line.

effects of the discharge current on the electrical characteristics and on the SW. The gas flow is fixed at 200 sccm (Standard Cubic Centimeter per Minute), whereas the gas pressure is constant and equal to $399 \mathrm{~Pa}$. Preliminary results obtained by using the Bari plasma facility with atomic and molecular gases are consistent with a thermal effect. At the same time, the shock initiation device in the present version of the tube has a low energy, therefore shock with Mach number only slightly larger than 1 has been obtained [17]. Besides, the absence of Langmuir probe and molecular spectroscopy as part of the diagnostics strongly limits the possibility to detect plasma effects. The work in progress is now aimed at redesigning and rebuilding the plasma tube, in order to increase the Mach number achievable by reducing the tube section and modifying the shock initiation device, while the implementation of more advanced diagnostic facilities will be performed afterwards.

\section{Plasma Tube Modeling}

A Particle in Cell - Monte Carlo (PIC-MC) program has been developed to treat the problem of charge separation under the conditions of plasma shock tubes, in order to evaluate the electric potential and field which arise due to the non-zero space charge across the shock in a weakly ionized gas. A practical method is proposed for the calculation of component separation since these tubes operate in the transition regime. The basic idea is that the nonlinear fluid dynamics of the main component is treated first, while the final determination of all components is made by using the linear Test Particle Monte Carlo (TPMC) method. The TPMC method has been used to solve the linear transport equation for minor species (including electrons and ions), which is obtained by setting the target distribution $f_{0}(r)$ to match locally the fluid dynamics results for the main component shock. This method is totally free of numerical diffusion and is of simpler implementation than DSMC approach inspite of its obvious advantages for the treatment of seeded atomic flows, namely the variance reduction in the treatment of the impurities and the expected good reliability of the calculated atomic separation in view of the lack of numerical diffusion.

The transport of more complex and structured seed species (e.g. molecules) should be possible by straight forward extensions of the linear part of the model. The use of linear transport methods well developed for thermal neutrons offers the possibility to borrow standard variance reduction techniques from this field [33, 34]. For example, if an estimate of the separation effect is needed in the front shock, it is possible to inject particles in the flow just before the shock and remove them just after it. In this way a drastic reduction of the computational cost is possible. A more advanced treatment can employ the adjoint equation in order to provide local solutions for a few space positions of interest.

Argon has been considered as discharge feed. The electric potential is the unknown of the Poisson equation on the numerical grid on which particles are sampled, coherently with the PIC-MC methodology, i.e.

$$
\begin{aligned}
& \nabla^{2} \varphi=-4 \pi \rho \\
& \rho=e \int f_{\text {ion }}(r, v) d^{3} v-e n_{e}(r),
\end{aligned}
$$


(a)

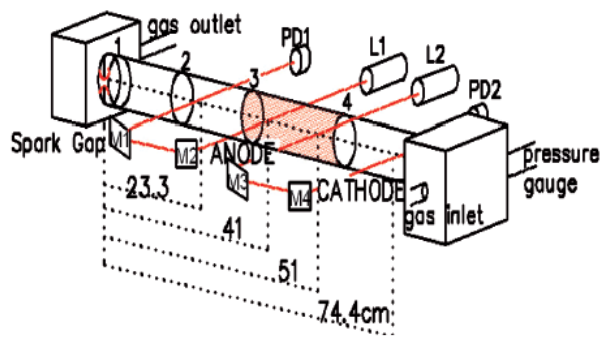

(b)

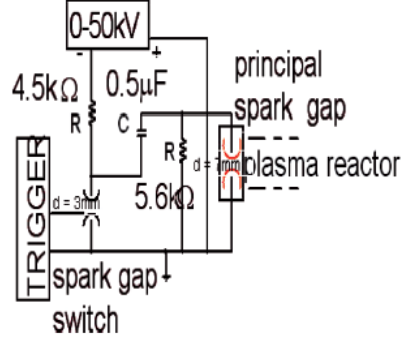

(c)

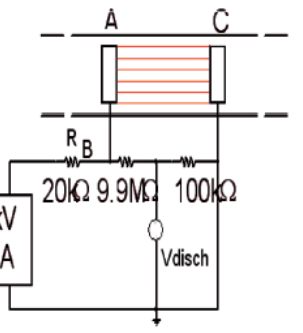

Fig. (3). Experimental set-up of the version of the Bari plasma shock tube used for the preliminary campaign of measurements (adapted from [17]): (a) Schematic of the apparatus; (b) Circuit diagram of spark gap; (c) Circuit diagram of DC discharge.

where $f_{\text {ion }}$ is the kinetic distribution of ions which is the result of the sampling of $\mathrm{MC}$ particles.

In the case of normal shocks a $1 \mathrm{D}$ model is to be developed (2D is expected for the case of plasma boundary layer); furthermore, in a first evaluation of the 1D code we concentrated on ion kinetics, since ions are mostly responsible for the plasma contribution to viscosity. The solution for electrons has been according strongly simplified as $n_{e}(x)=\alpha n_{A r}(x)$.
To calculate $\rho(x)$ we have to know the position of charged particles at every time, but the particle dynamics evolves in time through an appropriate time step $d t$, whereas the free flight times $t_{c}$ are random and vary for every particle. So to harmonize this different time period we have used the modified time step technique of Hockney and Eastwood. In a generic time iteration $d t$, the $i-t h$ particle undergoes a definite number of collisions. We introduce the residual of the time step $d t^{\prime}$, which we set initially equal to $d t$, and we select randomly the free flight times $t_{c}(i)$. If

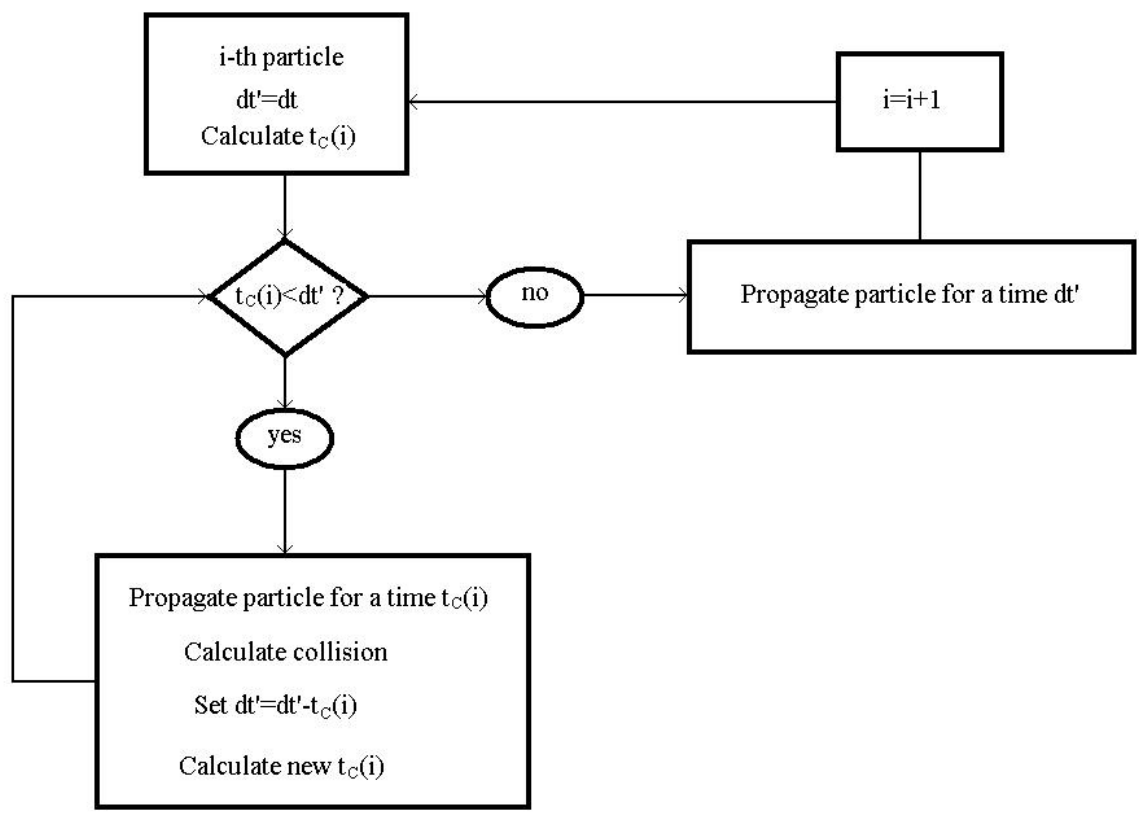

Fig. (4). Modified time step flow chart. 
$t_{c}(i) \leq d t^{\prime}$, the particle propagates freely and collides, then $d t^{\prime}$ is updated to $d t^{\prime}-t_{c}(i)$. Now a new value for $t_{c}(i)$ is selected, and the procedure begins again until $t_{c}(i) \geq d t^{\prime}$. In this case the collision do not occurs, but the i-esim particle undergoes a free propagation during the residual time $d t^{\prime}$. This algorithm is schematized in Fig. (4).

A first series of runs has been performed under the following conditions: $M=1.73, n_{A r}=3.22 \cdot 10^{23} \mathrm{~m}^{-3}$, $\alpha=10^{6}$, and $T=300 \mathrm{~K}$. The result of this first series of run are shown in Figs. (5-7). Fig. (5a) shows that a net charge density appears in the shock region, with a change of sign across the shock, and an apparent zero average across the whole shock. This behavior in our simple model is due to the diffusion of positive charges ahead of the shock, since the electrons are considered as a steady term. In real conditions the diffusion of electrons cannot be neglected, but our method can be still used with a much higher consumption of computer resources. This interpretation of the phenomenon is confirmed by Fig. (5b) and in Fig. (6) reporting the ion

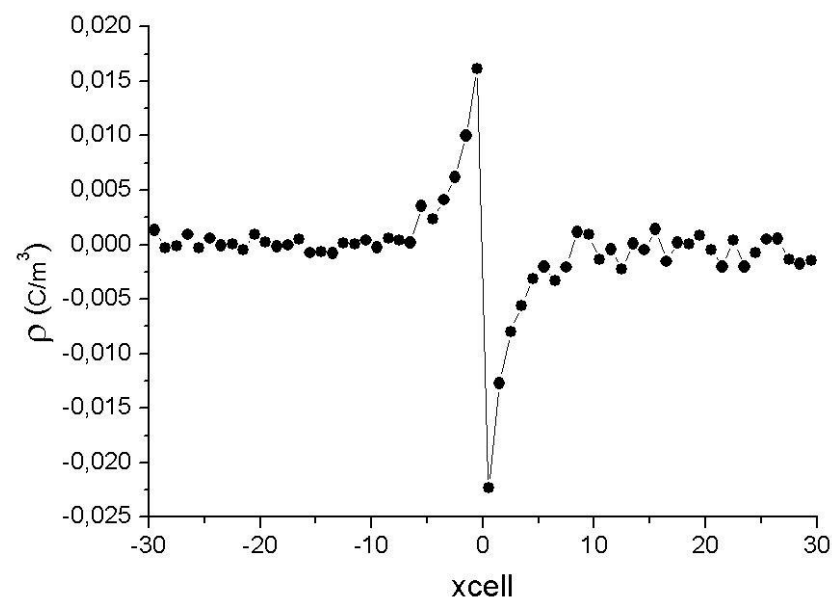

Fig. (5). MC simulation: (a) Space charge density, (b) Density.

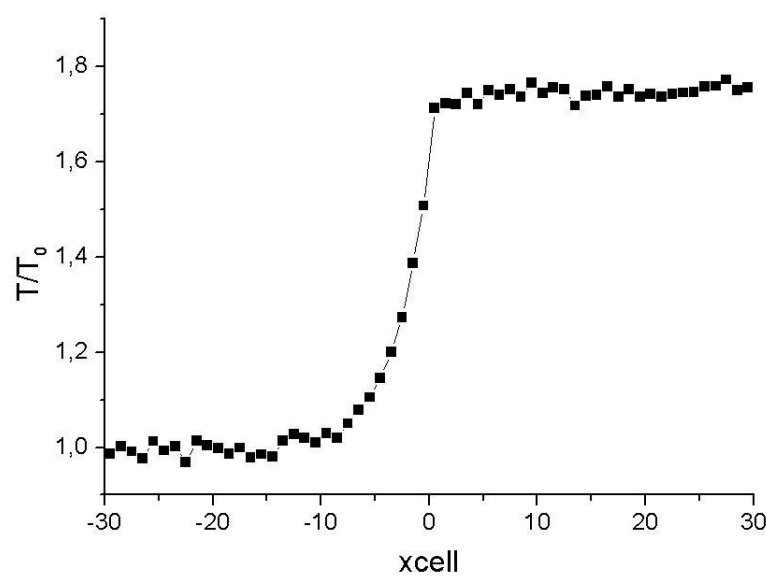

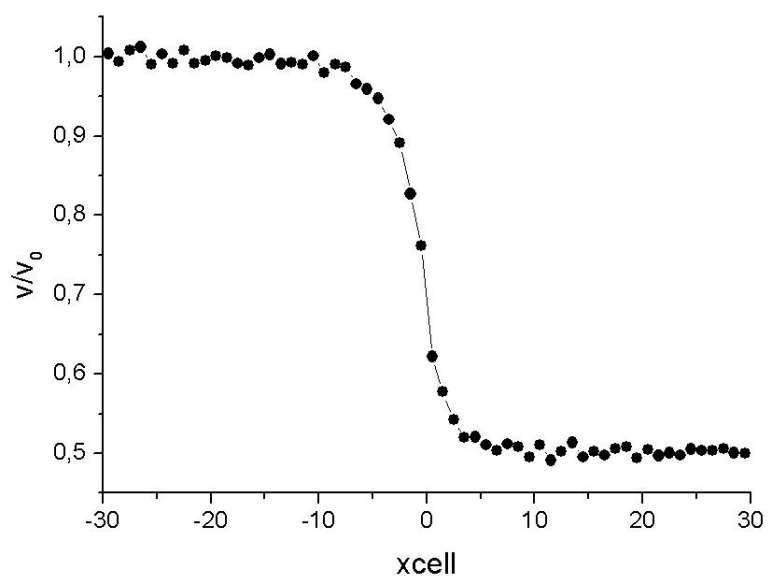

density and average speed respectively. In Fig. (7) the selfgenerated electric field is plotted.

As can be seen in Fig. (7), the pure collisional dynamics of ions in a shock front lead to the development of a residual electric field after the shock. This result of course is not physical and show that the ions and the electrons will be subjected to a self-generated field and consequently accelerated until the residual field is removed; This happens when the total charge across the shock is exactly neutralized. Therefore it is of interest to establish and study the effect of an electric field on the ion kinetics in a shock. Furthermore, in an experiment of shock propagation in a partially ionized gas, an electric field is already present, and such field can also be transversal i.e. not directed along the mean velocity in the flow. In view of the interest of such information, we have performed numerical experiments of ion propagation in the shock including either transversal or longitudinal electric fields.

The electric field effect is accounted during the free flight time based on the electric potential evaluated using the modified time step technique [35], where the particle dynamics of a simulated ensemble evolves in time with an

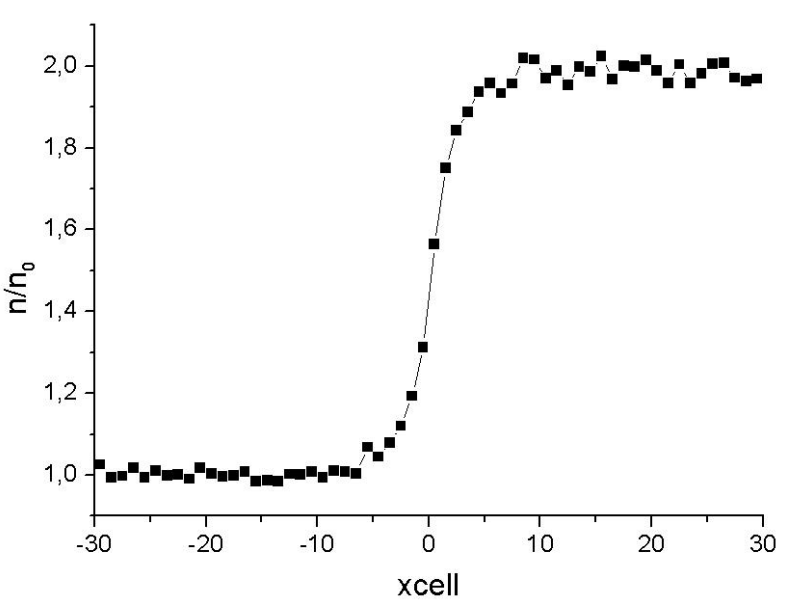

xcell

Fig. (6). MC simulation: (a) Temperature, (b) Velocity. 
appropriate time step while the time-to-next collision is an independent variable for any particle, which is gradually reduced during the free-flight. New free-flight time are generated after collision events. For the same reason, the inclusion of electric field gradients adds no special difficulty.

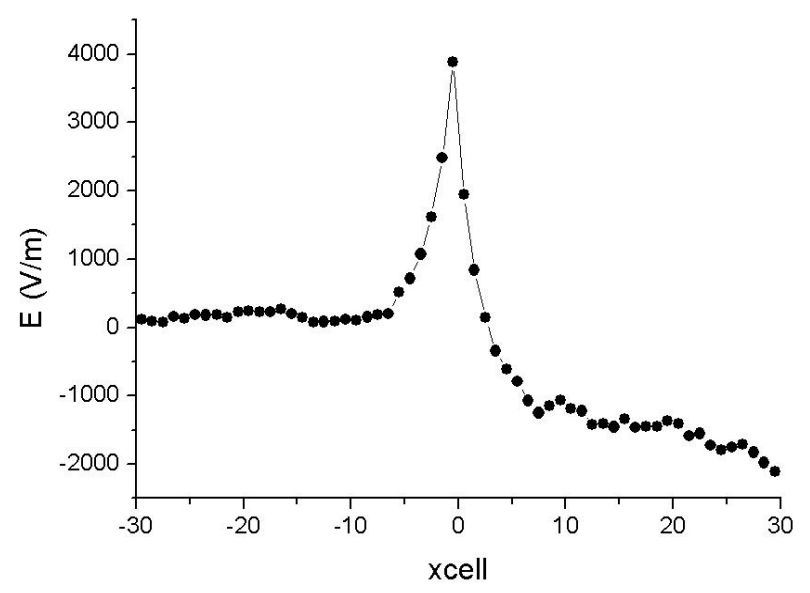

Fig. (7). Electric field intensity computed by MC simulation.

The effects are shown in Figs. $(\mathbf{8}, \mathbf{9})$ where relative ion concentration, ion temperature, and longitudinal component of ion velocity are represented. In Fig. (8b) we see that longitudinal and transversal electric field produce precursor heating of the ions ahead of the shock. Both the fields also produce a sharp increase in the ion temperature behind the shock. Much interesting, a temperature overshoot is observed which was absent in without the electric field. This overshoot is due to the fact that the ion directed speed is essentially determined from the reduced electric field $E / N$, which leads to a decrease of the electric heating in the post shock. The directed speed of the ions is changed to thermal energy when the neutral density increases, thereby producing a transient overheating. The effect is observed in both cases when the $E$ field is added. However, the effects produced by the two orientations of the field with the shock propagation directions are sensibly different in details. When the field $E$ is transversal, the temperature overshoot is higher; This effect cannot be separated from the clear higher temperature of the ions ahead of the shock, which is higher in the case of the transversal field. In the case of the longitudinal field this is directed towards the shock, and particles which are moving opposite to the flow have a much different translational distribution $f_{i o n}(v)$. When the particles cross the shock boundary, they not only find themselves in a denser environment, but also the flow speed is reduced. The consequent relaxation of $f_{\text {ion }}(v)$ accounts for the stronger $T$ overshoot. These preliminary results provide theoretical basis for limited but detectable plasma effects on shock propagation via electrical diagnostics and molecular spectroscopy, based on the electric field produced by the shock, the ion-molecule energy transfer and the excitation of internal levels. However, it must be noted that these calculations describe shock conditions which are not presently accessible, although they should be achieved using the improved version of the Bari plasma tube under design.

\section{DC-PLASMATRON SUBSONIC PLASMA JET}

\section{Experiments}

The DC-Plasmatron facility, built at the Institute of Electric Power Engineering at Poznan University of Technology and schemed in Fig (10), consists of a thoriated tungsten rod cathode of $6 \mathrm{~mm}$ diameter, placed in a watercooled housing and copper cylindrical sections with the internal diameter of $12 \mathrm{~mm}$ and $30 \mathrm{~mm}$ long. The sections are insulated by means of rings used to inject gas in such a way that a vortex develops in the plasma torch channel. This vortex movement is necessary to stabilize the plasma and to avoid a destruction of sections. Cathode, anode and other sections have a separated water cooling circuits, thus allowing evaluation of the energy exchanges. The plasmatron is mounted on a low pressure chamber $780 \mathrm{~mm}$ long with diameter of $100 \mathrm{~mm}$. The chamber is equipped
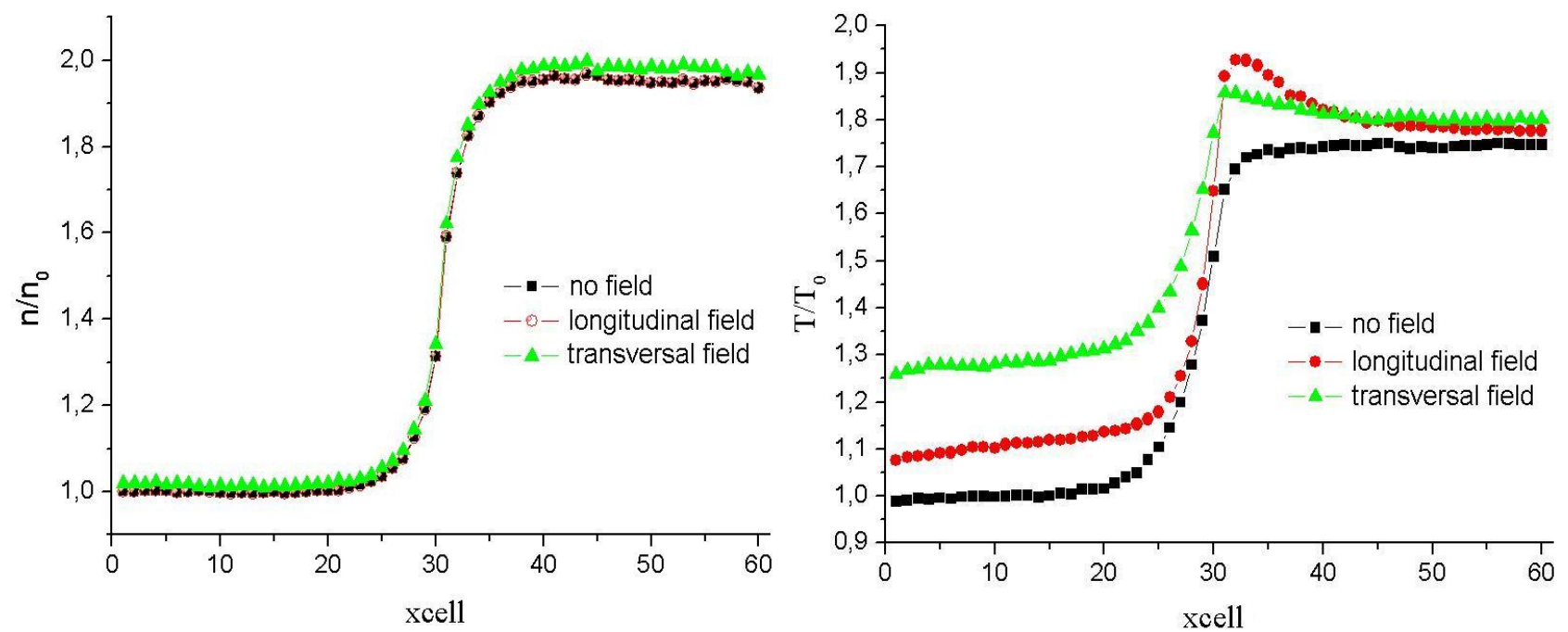

Fig. (8). MC simulation: (a) relative ion concentration, (b) ion temperature. 


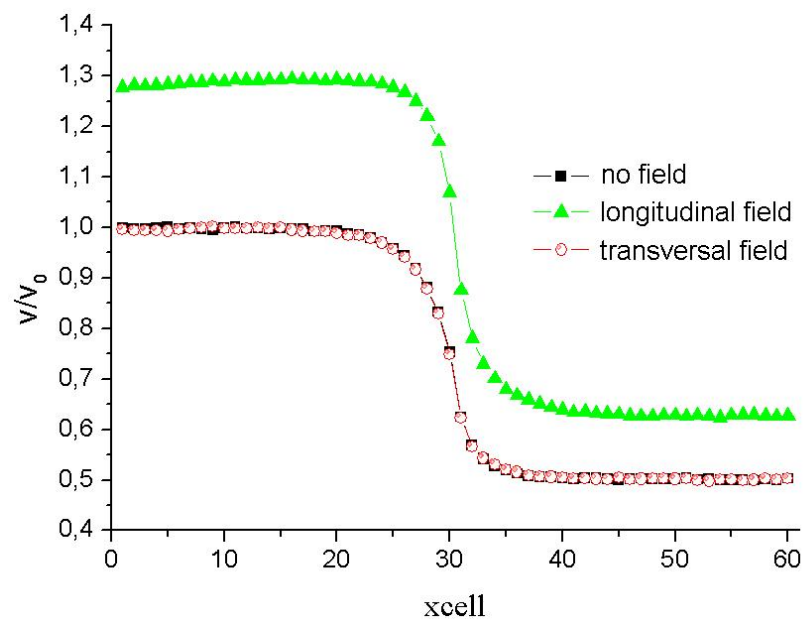

Fig. (9). Longitudinal component of ion velocity computed by the PIC-MC simulation.

with observation windows. The reduced pressure inside the chamber is achieved by two rotary vane pumps with electric power $1.5 \mathrm{~kW}$ each and Roots vacuum pump with a power of $4 \mathrm{~kW}$. The arc is ignited between the cathode and the first section serving as an anode using a high voltage-high frequency discharge. There is a possibility to add a second arc between two other segments in order to increase the electric input power. Two welding power sources connected in series are used to supply an electric arc. The experiments aim to study the emission of $\mathrm{N}$ and $\mathrm{C}$ atoms, $\mathrm{CN}, \mathrm{N}_{2}, \mathrm{~N}_{2}^{+}$ molecules in $\mathrm{N}_{2}-\mathrm{CO}_{2}$ mixture. For this study the nitrogen is injected into the arc, and behind the arc into the plasma jet nitrogen or $\mathrm{CO}_{2}$ is introduced. In this paper the measurements in pure nitrogen plasma flow are described therefore both in arc and plasma jet nitrogen gas is injected. Since the arc cannot be sustained in $\mathrm{CO}_{2}$ gas due to the torch construction in the next experiments with $\mathrm{N}_{2}-\mathrm{CO}_{2}$ mixture $\mathrm{CO}_{2}$ gas will be introduced into the plasma jet region. In this experiment the plasmatron consisted of single section as anode and only one after anode. During the experiment the plasmatron was working with arc current of $347 \mathrm{~A}$ and mean arc voltage about $50 \mathrm{~V}$ giving a delivered power of $17.4 \mathrm{~kW}$. The static pressure inside chamber was $55 \mathrm{~m}$ bar, gas flow rate injected into arc $0.8 \mathrm{~g} / \mathrm{s}$ and injected into plasma jet $0.6 \mathrm{~g} / \mathrm{s}$. Assuming the temperature of heavy particles $6500 \mathrm{~K}$ (see characterization hereafter), it gives the Mach number 0.6 (flow velocity was about $920 \mathrm{~m} / \mathrm{s}$ ). The emission spectra of the plasma jet are registered with the use of a spectrograph/monochromator (Acton, model SpectraPro2500i) and an ICCD camera (Andor, iStar). The spectrograph is equipped with three gratings 2400, 1800, and 600 grooves $/ \mathrm{mm}$. In the case of $2400 \mathrm{~g} / \mathrm{mm}$, the reciprocal dispersion in the visible region is $0.005-0.008 \mathrm{~nm} /$ pixel depending on the wavelength. The width of the entrance slit was $20-50 \mu \mathrm{m}$. The plasma is imaged on the entrance slit either using $180 \mathrm{~mm}$ achromatic lens (or fused silica $300 \mathrm{~mm}$ lens) mounted on a movable table. The entrance slit of the spectrograph is perpendicular to the direction of plasma expansion and its width and magnification determine the spatial resolution of the imaging system in this direction. The reduction of the plasma size on the entrance slit of the spectrograph is 6.25 (in the case of $180 \mathrm{~mm}$ lens). The spatial resolution along the slit is given by the CCD camera track width which was $260 \mu \mathrm{m}$, i.e. $1.6 \mathrm{~mm}$ including magnification. Such a configuration enables the Abel inversion of axially symmetric jet. The whole optical system was calibrated with the use of DH-2000-CAL Deuterium Tungsten Halogen Calibration Standard and in some spectral regions also with the use of a tungsten ribbon lamp. The spectra were registered at a distance of $65-95 \mathrm{~mm}$ from the anode.

\section{Results}

\subsection{Atomic Lines}

The emission spectra of nitrogen plasma consisted of atomic nitrogen lines and $\mathrm{N}_{2}^{+}$molecular bands. The lines of nitrogen were clearly distinguishable only in the infrared region. In other spectral region weak nitrogen lines were often overshadowed by the impurity W I lines. Although it was determined that the level of impurities usually did not exceed $0.001 \%$ of nitrogen, due to low excitation energy tungsten lines seriously affected the observed spectra in some regions. No lines were broadened over the measured apparatus broadening so determination of the electron

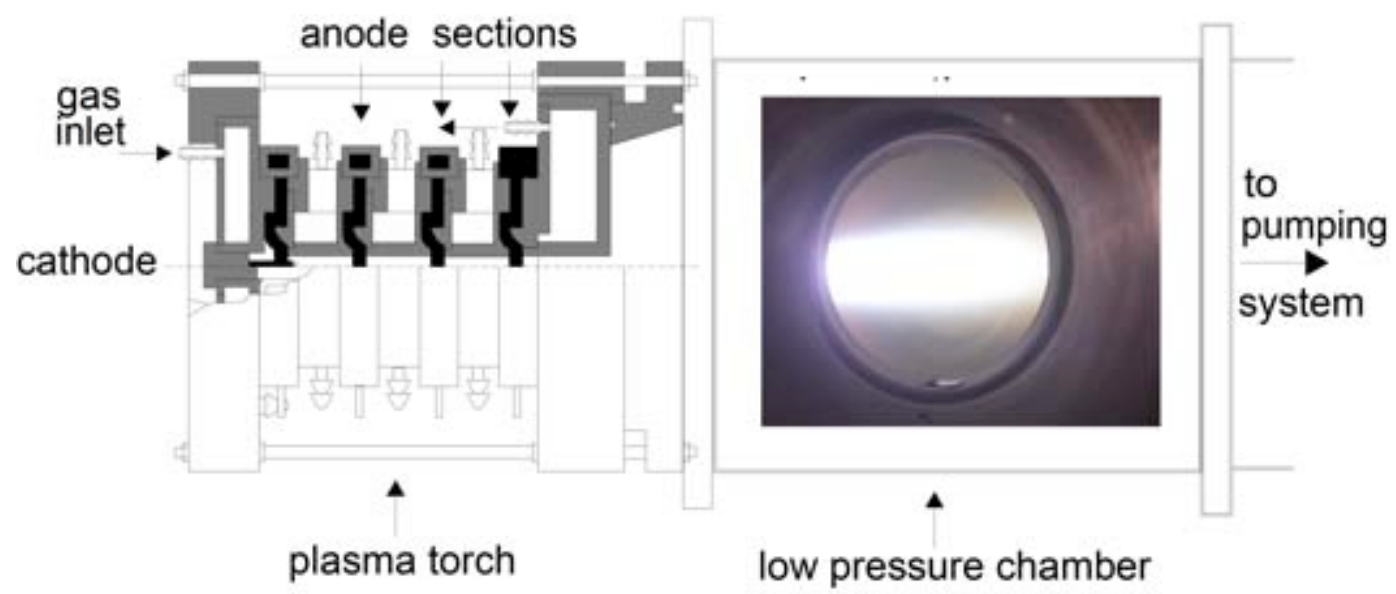

Fig. (10). Segmented plasma torch. 


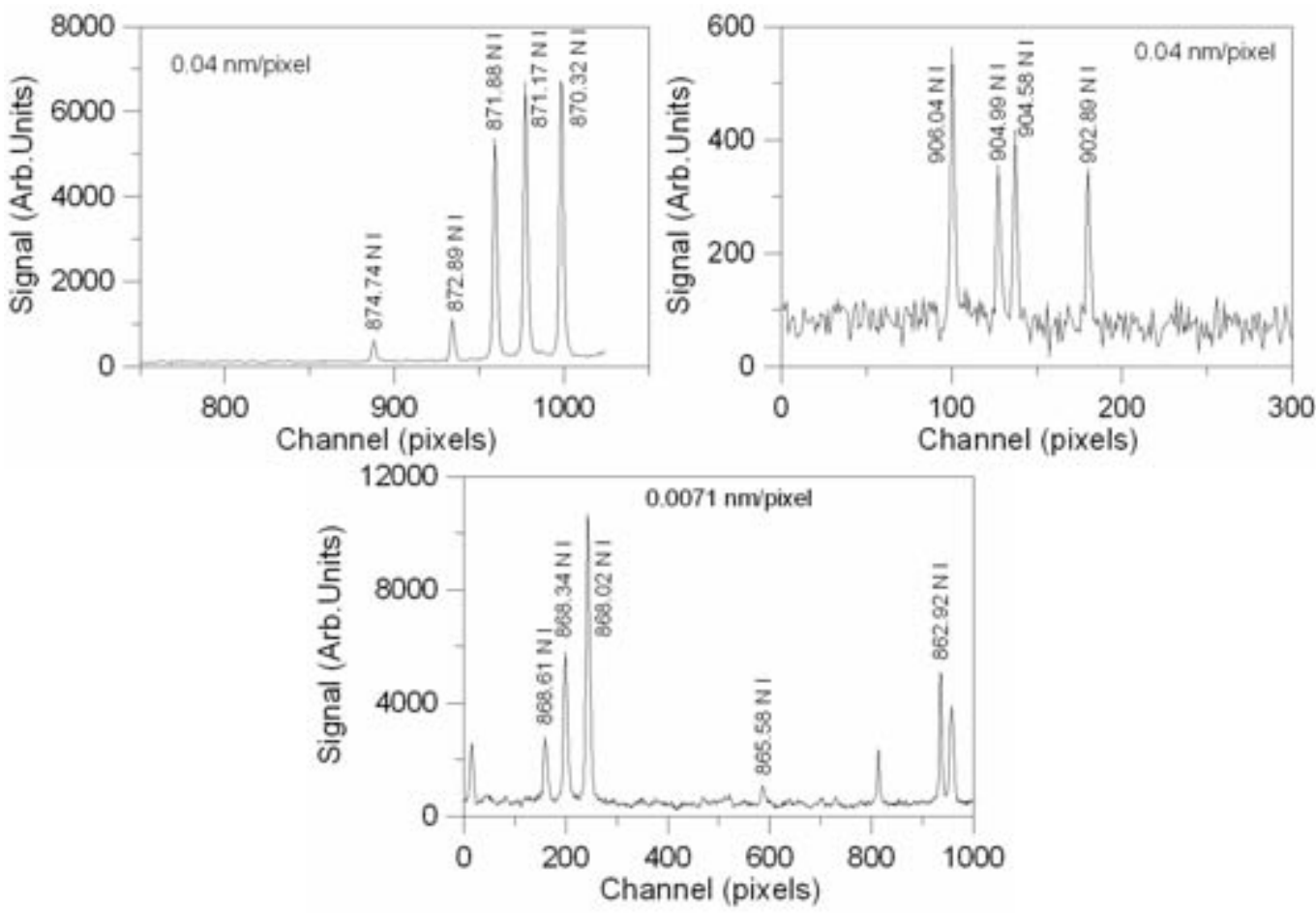

Fig. (11). Spectral lines of atomic nitrogen in infrared region.

density from the Stark broadening of spectral lines was not possible. Therefore only relative and absolute population densities could be determined from intensities of spectral lines. Some characteristic spectra of the atomic lines of nitrogen are shown in Fig. (11).

The excitation temperature $T_{\text {exc }}$ was determined from the relative intensities of the following N I lines: 870.32, 871.17, $871.88,872.89,874.74,902.89,904.58,904.99,906.04 \mathrm{~nm}$ (see Fig. 11). The upper levels of these lines have energy $11.75 \mathrm{eV}, 12.97 \mathrm{eV}$ and $13.72 \mathrm{eV}$. The energy gap between the levels is wide enough to determine $T_{e x c}$ with good accuracy and all these lines could be registered together with the grating $600 \mathrm{~g} / \mathrm{mm}$. The Boltzmann plot from intensities integrated along the central plasma chord $95 \mathrm{~mm}$ from the anode is shown in Fig. (12). The estimated accuracy is $\sim 15 \%$. Unfortunately the lines originating from the transitions from highly excited levels (902.89-906.04 nm) are very weak which results in poor accuracy of Abel inversion. As a result the radial distribution of the electron temperature is flat with $T_{e x c}$ about $8000 \mathrm{~K}$. The absolute calibration allows the determination of population densities of the upper levels of measured lines. It seems that all levels with energy $11.75 \mathrm{eV}$ or higher are in equilibrium with a $T_{e x c}$ of about $8000 \mathrm{~K}$. The population density of upper level of $746.83 \mathrm{~nm} \mathrm{~N}$ I line is shown in Fig. (12).

The measured lines were checked to be free from selfabsorption by examining the plasma optical depth for a spectral line using the formula [36] (in SI units)
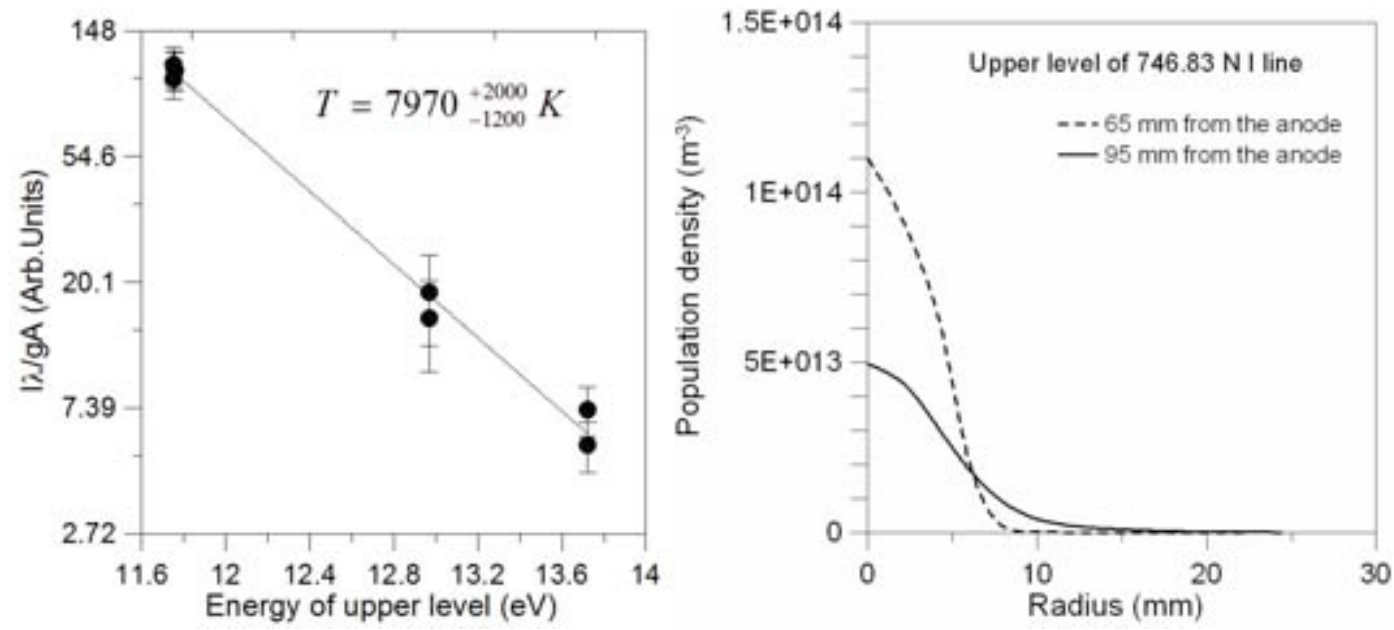

Fig. (12). At left, Boltzmann plot from relative N I lines intensities. At right, population density of upper level of $746.83 \mathrm{~nm} N \mathrm{I}$ line. 

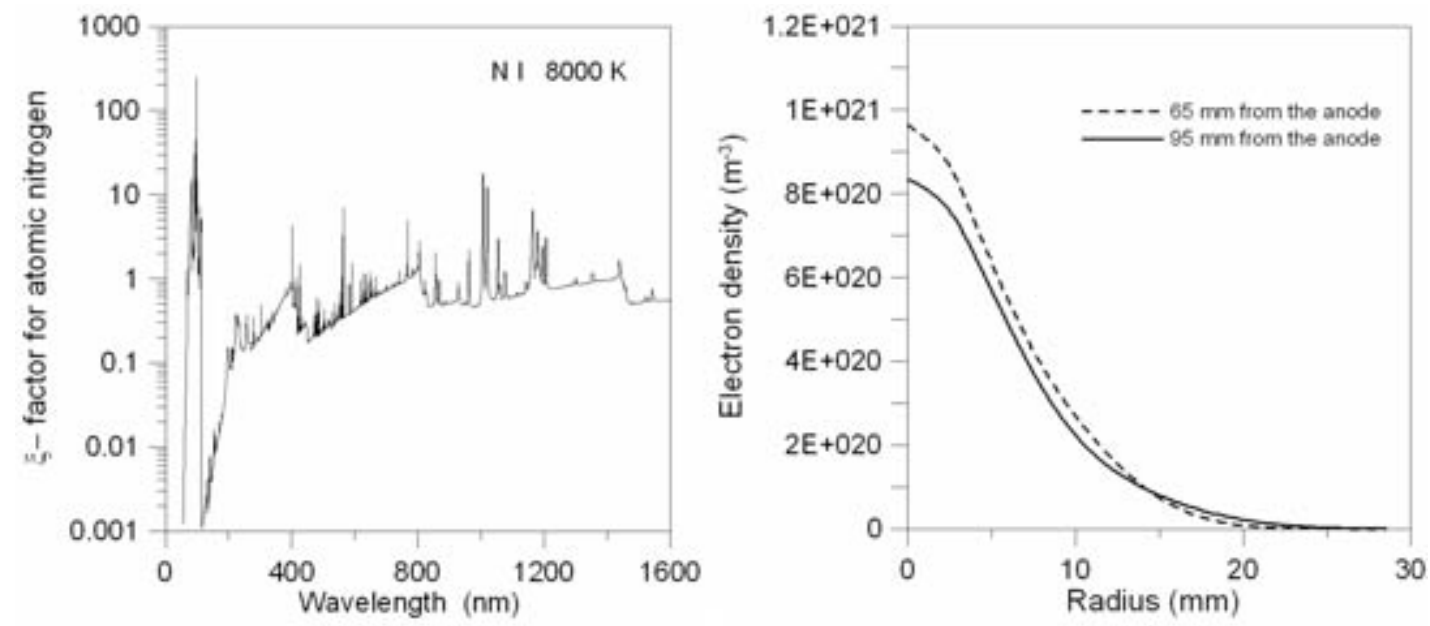

Fig. (13). At left, $\xi$ factor for atomic nitrogen at $T_{e}=8000 \mathrm{~K}$. At right, radial distributions of the electron density at two different distances from the anode.

$$
\tau_{k i}=8.85 \times 10^{-15} f_{i k} \times \lambda^{2} n_{z, i} d \times\left[1-\exp \left(-h c / \lambda k T_{e}\right)\right] P_{i}\left(\lambda_{0}\right)
$$

where $\tau_{k i}$ denotes the optical depth in a line centre, $f_{i k}$ the absorption oscillator strength, $\lambda$ the wavelength, $n_{z, i}$ the population density of the lower level, $d$ the plasma dimension, $h$ the Planck constant, $k$ Boltzmann constant, $c$ the speed of light in vacuum, $T_{e}$ the electron temperature and $P_{k i}\left(\lambda_{0}\right)$ the line profile in a line centre. For Gaussian profile $P_{k i}\left(\lambda_{0}\right)=\sqrt{\frac{\ln 2}{\pi}} \frac{1}{\Delta \lambda_{1 / 2}}$, where $\Delta \lambda_{1 / 2}$ is half-width at half-maximum (HWHM).

For a given line profile, the relation between the optical depth in a line centre and the total line absorption can be easily evaluated [36]. The absorption can be neglected when the optical depth in a line centre is lower than 0.1. The spectrometer was calibrated with the use of a tungsten ribbon lamp placed at the same distance as the plasma. The calibration allowed us to determine the population densities of the upper levels of spectral lines used in the experiment. Next, the population densities of the lower levels were obtained assuming the Boltzmann distribution between upper and lower level. The half-widths of the lines were determined mainly by the Doppler broadening. The results showed that the lines used for the electron temperature determination were not influenced by self-absorption.

\subsection{Continuum Radiation}

Since the Stark broadening of spectral lines was too small the electron density was determined from the continuum radiation. The method does not require thermodynamic equilibrium to be valid. The total continuum emission coefficient can be written as

$\varepsilon_{\text {total }}=\varepsilon_{e i}^{f b}(\lambda)+\varepsilon_{e i}^{f f}(\lambda)+\varepsilon_{e a}^{f f}(\lambda)$,

where $f b$ and $f f$ indices denote free-bound and free-free transitions i.e., the emission due to the recombination and the Bremsstrahlung respectively and $e i$ and $e a$ denote electron- ion and electron-atom collisions. Formulas for $\varepsilon(\lambda)$ are written below (in SI units).

$$
\begin{aligned}
& \varepsilon^{f b}(\lambda)=1.632 \times 10^{-43} N_{e} T_{e}^{-1 / 2} \lambda^{-2} \frac{g_{z, 1}}{U_{z}}\left(1-\exp \left\{\frac{h c}{\lambda k T_{e}}\right\}\right) \\
& \sum_{z} N_{z} z^{2} \xi\left(T_{e}, \lambda\right) \\
& \varepsilon^{f b}(\lambda)=1.632 \times 10^{-43} N_{e} T_{e}^{-1 / 2} \lambda^{-2} \frac{g_{z, 1}}{U_{z}} \exp \left\{-\frac{h c}{\lambda k T_{e}}\right\} . \\
& \sum_{z} N_{z} z^{2} G^{f f}\left(T_{e}, \lambda\right) \\
& \varepsilon_{(e a, \lambda)}=1.026 \times 10^{-34} N_{a} N_{e} \lambda^{-2} T_{e}^{3 / 2} \sigma_{e a}(T) \\
& {\left[\begin{array}{l}
\left.1+\left(1+\frac{h c}{\lambda k T_{e}}\right)^{2}\right] \\
\exp \left(-\frac{h c}{\lambda k T}\right)
\end{array}\right.}
\end{aligned}
$$

where $N_{e}, N_{z}$ and $N_{a}$ is the electron, ion and atom density, respectively, $z$ denotes ion charge seen by free electron, $U_{z}$ is the ion partition function, $g_{z}$ is the statistical weight of the ion, $G^{f f}$ the Gaunt factor, $\xi$ is a correction (Biberman) factor [37], and $\sigma_{e a}$ is the cross section for electron-atom collisions. The formula (4) with $\xi=1$ has been derived for hydrogen-like atoms and the $\xi$-factor introduces the edge structure of the real coefficients [37]. The $\xi$ factor for nitrogen atom was calculated with the use of photoionization cross sections taken from [38]. It has been assumed that $T_{e}=T_{e x c}$. The Gaunt factor was taken from [39]. The formulas (4) and (5) were taken from [37] and formula (6) from [40]. All above formulas can be found in [41]. The formula (6) was also used for electron- $\mathrm{N}_{2}$ collisions. The cross sections for $\mathrm{e}-\mathrm{N}$ and $\mathrm{e}-\mathrm{N}_{2}$ collisions were taken from [42]. It has been found that in present experiment the continuum radiation due to recombination is 
dominant, other processes did not exceed $8 \%$ of total radiation. The radial distributions of the electron density calculated from the continuum radiation at $\sim 430 \mathrm{~nm}$ are shown in Fig. (13).

\subsection{Molecular Spectra}

The observed molecular spectra consisted mainly from the 1st negative system of $\mathrm{N}_{2}^{+}$. The rotational and vibrational temperatures were determined by comparing synthetic spectra issued from the SPARTAN code of M. Lino daSilva [43] with spectroscopic measurements. Both spectra are shown in Fig. (14). The best fit is obtained for rotational temperature $T_{R}=6500 \mathrm{~K}$ and vibrational temperature $T_{V}=10000 \mathrm{~K}$ indicating a non-equilibrium plasma flow.

Assuming that $T_{R}$ is equal to the atom temperature and taking into account that the flow velocity was about $920 \mathrm{~m} / \mathrm{s}$ we get the flow Mach number of about 0.6.

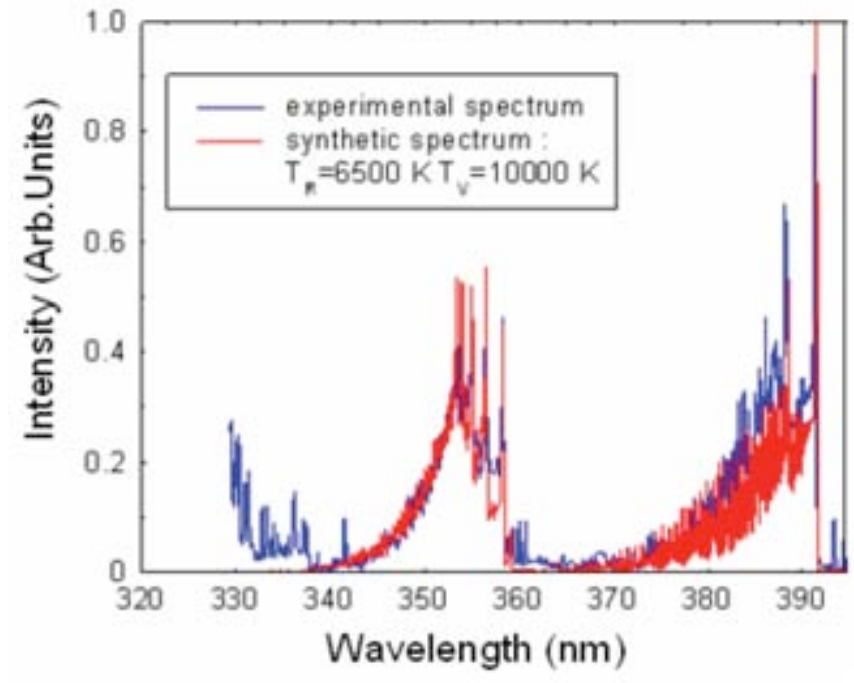

Fig. (14). Experimental and synthetic spectra of $\mathrm{N}_{2}^{+}$molecular bands at a distance $95 \mathrm{~mm}$ from the anode.

\section{Concluding Remarks}

The results show that the plasma produced by the segmented plasmatron is in non-equilibrium. This is what can be expected. The criterion for local thermodynamic equilibrium (LTE) has the form [44]:

$N_{e} \geq N_{e}^{c r}=1.6 \times 10^{18} T_{e}^{\frac{1}{2}}(\Delta E)^{3}$

where $N_{e}^{c r}$ is the critical electron density (in $\mathrm{m}^{-3}$ ) necessary to fulfil LTE conditions and $\Delta E$ is the largest energy gap of atomic (ionic) energy level system (in eV). Since such energy gap for N I is $10.33 \mathrm{eV}$ (the level with energy 3.55 $\mathrm{eV}$ has been neglected in these considerations because the transition rate to this level is weak) the electron density necessary to fulfill the above criterion is $\sim 1.6 \times 10^{23} \mathrm{~m}^{-3}$ at a temperature of $8000 \mathrm{~K}$. Even taking into account that in practice this criterion can be lowered one order of magnitude because of the absorption of resonance lines the electron density necessary to fulfill LTE conditions is still 10 times higher than that observed in the experiment. In addition the transport of particles can also influence the Saha balance. To summarize, the results represent subsonic nitrogen plasma in strong non-equillibrium which can be used to test the validity of collisional-radiative models.

\section{RF-PLASMATRON SUPERSONIC PLASMA JET}

\section{Experiments}

The experiments described hereafter have been performed with the PWT facility so-called VKI-Plasmatron. The Plasmatron facility includes an Inductively Coupled Plasma torch using a single-turn inductor powered by a high frequency, high power, and high voltage $(400 \mathrm{kHz}, 1.2 \mathrm{MW}$, $2 \mathrm{kV}$ ) solid state (MOS technology) generator. The discharge is generated inside the torch composed of a quartz tube with an internal diameter of $200 \mathrm{~mm}$ and $5 \mathrm{~mm}$ thickness, and also a cold cage of $160 \mathrm{~mm}$ internal diameter, made of water-cooled copper segments, inserted inside to prevent from severe overheating. The test gas, which is ambient air in the experiments reported here, is injected inside the torch with an annular injection. Once the plasma is started, the electric discharge is enhanced mainly by induced azimuthal electronic currents. The ionized flow is steadily heated by Joule effect, due to efficient electron-neutral energy transfers, absorbing RF power penetrating the plasma over the resistive skin depth, also called the Kelvin length. In the present experiments, the Plasmatron is operated at $490 \mathrm{~kW}$ power and $4.5 \mathrm{~g} / \mathrm{s}$ mass flow rate settings. Supersonic plasma jet is obtained by expanding the plasma through a strictly convergent nozzle (sonic nozzle) of $35 \mathrm{~mm}$ throat exit diameter into the test chamber where a low pressure is maintained. The pressure in the reservoir $P_{\text {res }}$ was set to 120 mbar and the supersonic plasma jet was investigated with chamber pressure $P_{c}$ set to $12 \mathrm{mbar}$ and to $6 \mathrm{mbar}$, corresponding respectively to moderately and highly underexpanded plasma jet cases. Images of the plasma jet acquired with a High Speed Camera (HSC) are displayed in Fig. (15) to visualize the general aspect of the supersonic jet. The underexpanding jet is typically characterized by a pressure at the nozzle exit higher than in the chamber. In order to equilibrate the pressure difference at the nozzle exit, expansion waves originating from the Prandtl-Meyer expansion are directed towards the jet axis and then reflected toward plasma jet boundaries. The edges of the expansion zone are bright consequently to the reflection of the expanding flow by the cold dense surrounding gas, giving rise to the so-called Barrel shocks. In our conditions, a well distinguishable Mach disk takes place downstream of the first expansion cell. The post Mach disk region is characterized by subsonic velocities together with an increase of the local temperature and pressure typical of post shock relaxing flows. Abel transformation applied to HSC image, also evinced in Fig. (15) gives insight into actual flow peculiarities such as the significant lack of emission in central regions of the jet consequently to the expansion (well pronounced at $P_{c}=6 \mathrm{mbar}$ ). 

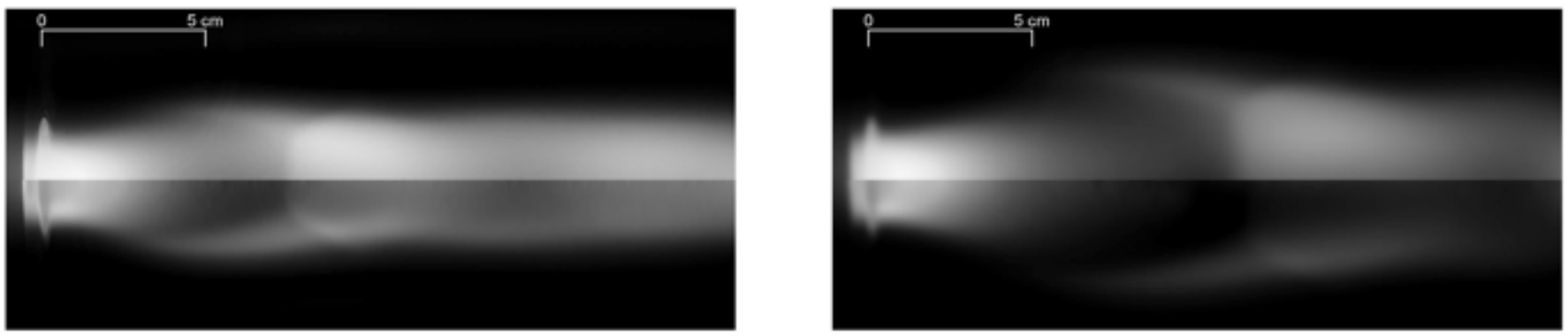

Fig. (15). HSC images for $P_{c}=12 \mathrm{mbar}$ (on left) and for $P_{c}=6 \mathrm{mbar}$ (on right) cases. The upper half is the actual record and the lower half is the one processed with Abel transform.

The plasma jet is characterized in terms of excitation temperature of atomic electronic states and electron density by means of Optical Emission Spectroscopy. The plasma jet line-of-sight emission is collected through an aperture and imaged with a fused silica lens onto the entrance slit of an Acton Series monochromator of $750 \mathrm{~mm}$ focal length. The spectrometer is combined with a ICCD PI-MAX camera, well-suited to deal with low intensity levels emitted by the rarefied region and offering a large recording frame of $1024 \times 1024$ pixels of $12.8 \mu \mathrm{m}$ side. The optical arrangement sketched in Fig. (16) was designed to obtain a radial slice of about $19 \mathrm{~cm}$ height optically conjugated with the CCD height with a magnification factor of 0.07 (the monochromator magnification being 1), in such a way the whole radial emission profile is recorded with the CCD frame with a spatial resolution of about $180 \mu \mathrm{m}$.

Plasma radiative signatures recorded in the range 400$900 \mathrm{~nm}$ are principally constituted of atomic nitrogen and oxygen lines as illustrated in Fig. (16). Atomic line emission was recorded using a 150 groves $/ \mathrm{mm}$ grating. The presence of trace of water vapor in the initial cold air mixture resulted in the presence of hydrogen Balmer lines $\mathrm{H}_{\alpha}$ and $\mathrm{H}_{\beta}$ that have been recorded with a 1200 groves $/ \mathrm{mm}$ grating in order to resolve the Stark broadening. The spectral resolutions corresponding to both gratings were measured to be respectively of $0.5 \mathrm{~nm}$ and $0.06 \mathrm{~nm}$, with the monochromator entrance slit width set to $3 \mu \mathrm{m}$. The overall experimental setup is schemed in Fig. (16). The spectra recorded in the visible spectral range were calibrated in absolute intensity with a ribbon tungsten lamp and its documented absolute radiance (note that only relative intensity are reported in Fig. (17)).

Measured intensities were spatially filtered with a Butterworth-like digital filter. We checked that the plasma is fairly axisymmetric and the considered atomic transitions are optically thin within covered pressure and temperature ranges. Before performing the plasma characterization, the line of sight intensities $I_{\lambda}\left(\mathrm{W} . \mathrm{cm}^{-2} . \mathrm{nm}^{-1} \cdot \mathrm{sr}^{-1}\right)$ have been processed by mean of the Abel transformation to rebuild spectral distribution of local emission $\varepsilon_{\lambda}\left(\mathrm{W} . \mathrm{cm}^{-3} \cdot \mathrm{nm}^{-1} \cdot \mathrm{sr}^{-1}\right)$.

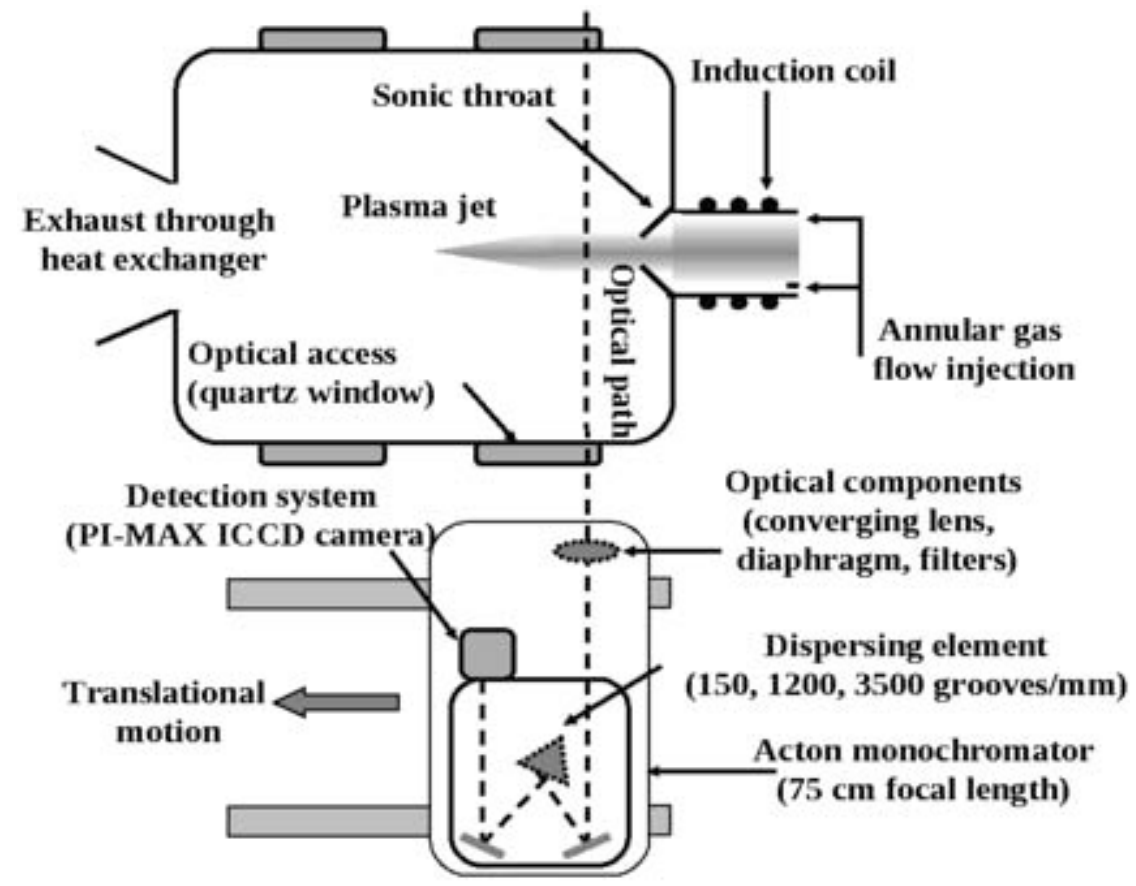

Fig. (16). Top view scheme of the experimental arrangement. 


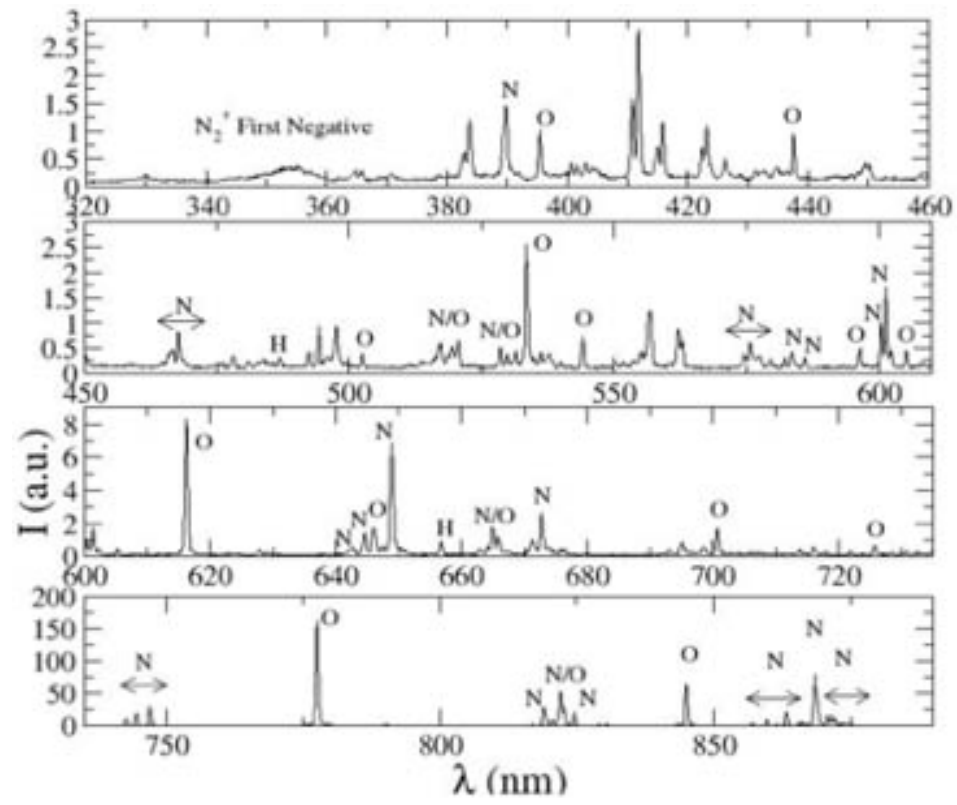

Fig. (17). Typical radiative signature for supersonic air plasma jet in the expansion region (case $P_{c}=12 \mathrm{mbar}$ ).

The optical arrangement was set on a translational stage in order to record radial plasma jet line-of-sight intensity at various positions along the jet $z$-axis. Intensity measurements started at $z=2.5 \mathrm{~cm}$ away from the nozzle exit, corresponding to the nearest axial location accessible by the optical access. Translating the optical arrangement along $z$-axis, radial distribution of plasma spectral intensity was measured at a dozen of axial locations to cover the first expansion, the Mach disk and the post shock regions. For moderately underexpanded jet $\left(P_{r e s}=120\right.$ mbar, $P_{c}=12$ mbar $)$, OES measurements were taken after nozzle exit at $2.5,3.5,4.5$, $6.5,7.5,8.5,9.5,10.5,11.5$ and $12.5 \mathrm{~cm}$. For highly underexpanded jet $\left(P_{\text {res }}=120 \mathrm{mbar}, P_{c}=6 \mathrm{mbar}\right)$, OES measurements were taken after nozzle exit at $2.5,3.5,4.5$, $8.5,10.5,11.5,12.5,13.5,15.5$ and $17.5 \mathrm{~cm}$.

\section{Characterization Methods}

\subsection{Electronic Excitation Temperature}

The characterization in terms of excitation temperature $T_{e x}$ is performed using measured local emission values $\varepsilon_{u l}$ associated to atomic radiative transition due to spontaneous emission from an upper (emitting) level $u$ to a lower level $l$. Knowing the emission $\varepsilon_{u l}$ allows a straight probing of the upper (emitting) level $u$ population density through the relation:

$\varepsilon_{u l}=n_{u} \frac{A_{u l}}{4 \pi} \frac{h c}{\lambda_{u l}} \quad\left(W \cdot m^{-3} \cdot s r^{-1}\right)$,

where $A_{u l}\left(\mathrm{~s}^{-1}\right)$ is the Einstein coefficient associated to spontaneous emission from level $u$ to level $l$, respectively of energy $E_{u}$ and $E_{l}\left(\mathrm{~cm}^{-1}\right)$ and $\lambda_{u l}(\mathrm{~nm})$ designates the line spectral position. The population of a given emitting level $n_{u}$ is determined through the spectral integration of its associated emission profile: $\varepsilon_{u l}=\int \varepsilon_{\lambda} d \lambda$, with $\varepsilon_{\lambda}$ referring to the spectral line profile of local emission corrected from a linear background adjusted on the line profile edges.

The excitation temperature $T_{e x}$ is evaluated on the basis of the Boltzmann diagram method, assuming that population of excited states $n_{i}$ is ruled by the Boltzmann distribution expressed as:

$n_{i}\left(T_{e x}\right)=\frac{N}{Q_{\text {int }}} g_{i} \exp \left(\frac{h c E_{i}}{k_{B} T_{e x}}\right) \quad\left(m^{-3}\right)$,

with $N\left(\mathrm{~m}^{-3}\right), Q_{i n t}$ and $g_{i}$ designating respectively the species concentration, the internal partition function and the degeneracy of level $i$. Starting from Eqs. (8) and (9), the measured density population versus energy $E_{u}$ can be expressed as:

$\ln \left(\frac{n_{u}}{g_{u}}\right)=-\left(\frac{h c}{k_{b} T_{e x}}\right) E_{u}+\ln \left(\frac{N}{Q_{\text {int }}}\right)$.

$T_{e x}$ is then simply obtained by mean of a straight line fitting on distribution of measured population densities. Required spectroscopic data for $T_{e x}$ determination were taken from NIST [45]. Uncertainties associated to this approach might be roughly evaluated as $\frac{\Delta T_{e x}}{T_{e x}} \approx 20 T_{e x} /\left(1.44 \times \Delta E_{\max }\right)$, where a critical error of $20 \%$ on local emission is assumed and $\Delta E_{\max }$ (expressed in $\mathrm{cm}^{-1}$ ) is the difference between upper energy level extrema. Hence, for a typical $\Delta E_{\max }$ of 
$\sim 25000 \mathrm{~cm}^{-1}$ and temperature of $10000 \mathrm{~K}$ the uncertainty is of $\sim 5 \%$.

For brevity, the present study considers only oxygen lines to evaluate the plasma excitation temperature, nitrogen lines are fewer and cover a $E_{i}=109837.02$ more reduced energy range making them less reliable than oxygen lines. The numerous atomic oxygen lines, taken into account in the $T_{e x}$ determination method, belong to three groups of transitions. Transitions $3 p \rightarrow 3 s$ of upper energy level below $90000 \mathrm{~cm}^{-1}$, including the triplet oxygen lines located at roughly $777 \mathrm{~nm}$, the group of transitions $7 s, 6 s, 5 d, 4 d, 5 s \rightarrow 3 p$ characterized by upper energy level $E_{u}$ ranging from 98000 to $107000 \mathrm{~cm}^{-1}$, and the transition located at $715.67 \mathrm{~nm}$ of upper energy level $E_{u}=116631.094 \mathrm{~cm}^{-1}$ above the first ionization energy $E_{\mathrm{i}}=109837.02 \mathrm{~cm}^{-1}$.

Typical Boltzmann diagram plots, presented in Fig. (18), show that at the early expansion, excited state population densities are slightly scattered and fairly distributed along a straight line. However their distribution might deviate significantly from a strict Boltzmann distribution in shock regions. In particular the population of the highest upper energy levels, located beyond the first ionization energy, was found to be systematically slightly underpopulated. In regions of low intensity, the temperature reliability is affected by the rise of the signal to noise ratio and the vanishing of lines of higher upper energy level.

\subsection{Electron Density}

The determination of the electron density $n_{e}$ is performed in a conventional manner by means of recorded hydrogen line profile fits with computed Stark broadened profiles. We used the up-to-date database proposed in [46] to model hydrogen $H_{\alpha}$ and $H_{\beta}$ line profile, located respectively at $656.279 \mathrm{~nm}$ and $486.135 \mathrm{~nm}$, undergoing broadening due to coulombian collisions. The database contains intensity profiles tabulated against wavelengths for several values of electron temperature $T_{e}$ and electron density $n_{e}$, covering respectively the range $10^{3}<T_{e}<10^{6} \mathrm{~K}$ and $10^{14} \leq n_{e} \leq 10^{19} \mathrm{~cm}^{-3}$. The computation of hydrogen line profile at any electron temperature $T_{e}$ and electron density $n_{e}$ is performed by mean of a bilinear interpolation of tabulated data. The computed Stark profile is then convoluted with the instrumental apparatus function.

Systematic computations have shown that computed line profile shapes exhibit a very weak sensitivity to the electron temperature in the range covered by the present experiments $(\sim 5000-15000 \mathrm{~K})$, while a slight electron density variation induces detectable modifications of the profile at our spectral resolution. Hence, the fitting procedure was performed with the electron density being the only unknown variable, the electron temperature being set to $8000 \mathrm{~K}$. The electron density $n_{e}$ is then obtained through minimization of the functional expressed as the Root Mean Square Error (RMSE) between the experimental and computed profiles:

$$
\operatorname{RMSE}\left(n_{e}\right)=\sqrt{\frac{1}{N} \sum_{i}^{N}\left(\overline{\varepsilon_{\lambda_{i}}}-\overline{\varepsilon_{\lambda_{i}}}{ }^{\operatorname{sim}}\left(n_{e}\right)\right)^{2}},
$$

where $N$ is the number of spectral grid points, $\overline{\varepsilon_{\lambda_{i}}}$ sim and $\overline{\varepsilon_{\lambda_{i}}}$ are the values at $\lambda_{i}$ of simulated and experimental line profiles scaled to unity. Typical $H_{\alpha}$ and $H_{\beta}$ measured spectral profiles are plotted in Fig. (18) together with their respective best fitted profiles, the one minimizing the RMSE value as defined above. Although the actual $H_{\beta}$ profile can be strongly overlapped with various other contributions, the fitting demonstrates a good tolerance and typically yields squared correlation coefficient value of $R^{2} \sim 0.98$.

\section{Results and Discussions}

Resulting $n_{e}$ and $T_{e x}$ radial profiles, obtained scanning axially the jet, have been appropriately arranged and
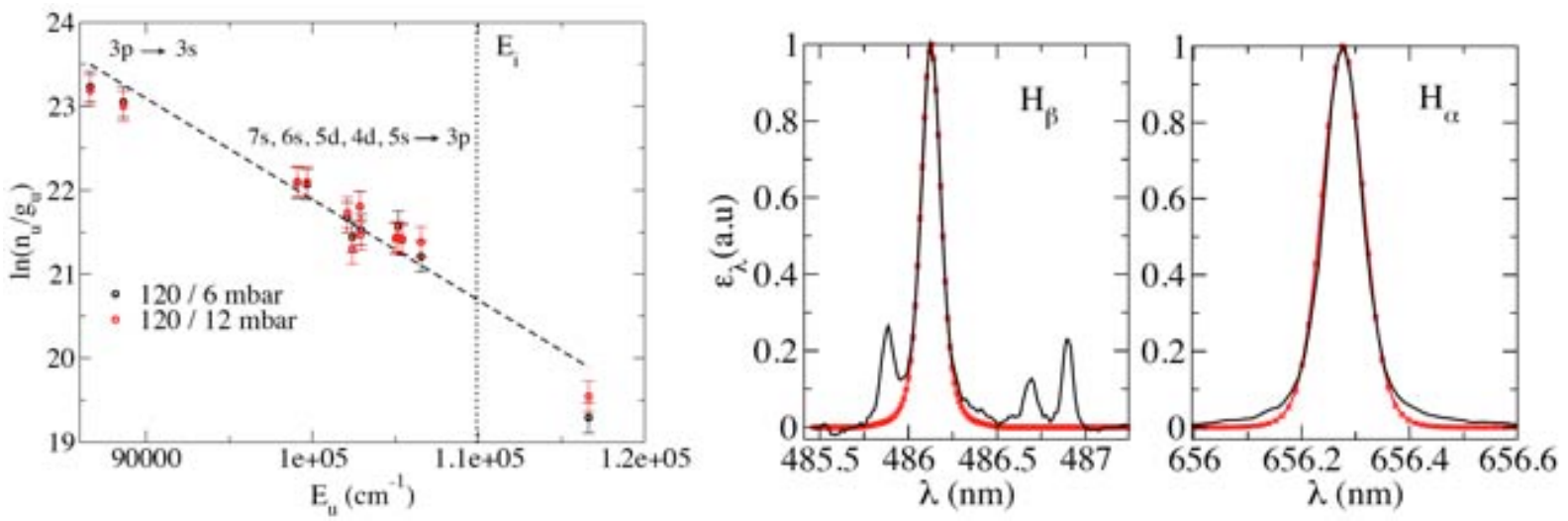

Fig. (18). Typical Boltzmann diagram plot at $z=2.5 \mathrm{~cm}$; black- $P_{c}=6$ mbar; red- $P_{c}=12$ mbar (on left). Typical best fits obtained with $\mathrm{H}_{\alpha}$ and $\mathrm{H}_{\beta}$ experimental line profiles (on right). 
processed to yield two dimensional distribution by means of bicubic spline interpolation under MATLAB environment. The 2D distribution of plasma emission in the visible spectral range is considered first. In Fig. (19), are displayed the resulting interpolations of the emission rebuilt by mean of Abel transform and integrated in the spectral range 400-900 nm (largely dominated by atomic emission).

The black lines correspond to the actual emission profiles taken into account by the interpolation procedure. Starting from the resulting emission 2D distribution, the Mach disk location is roughly gauged as the location at the end of the first expansion where the centerline emission starts rising. The estimated Mach disk position is $z_{M} \approx 7.6 \mathrm{~cm}$ at $p_{c} \approx 12$ mbar and $z_{M} \approx 11.5 \mathrm{~cm}$ at 6 mbar. Using the wellknown empirical correlation proposed in [47] in the case of an ideal gas expansion, the Mach disk position is expressed as:

$$
\frac{z_{M}}{D_{0}}=0.67 \sqrt{\frac{P_{c}}{P_{r e s}}},
$$

yielding in our experimental conditions $z_{M}=7.4 \mathrm{~cm}$ at $P_{c}=12 \mathrm{mbar}$ and $z_{M}=10.5 \mathrm{~cm}$ at $P_{c}=6 \mathrm{mbar}$, with nozzle diameter $D_{0}=3.5 \mathrm{~cm}$. Although the experimental estimations and the empirical values are in satisfactory agreement, the discrepancies could be attributed to the non ideal gas effects.

The interpolated distributions for $n_{e}$ are presented in Fig. (20). The black lines indicate the actual radial extent of $n_{e}$ measurements derived from $H_{\beta}$ line profiles. Density lower than $1.10^{14} \mathrm{~cm}^{-3}$ were not measured with respect to the validity domain of Stark broadening prediction [46]. After the first expansion region, the $n_{e}$ distribution is greatly influenced by the chamber pressure, while the electron density range is slighter affected after the nozzle exit. The electron density drops rapidly after the nozzle exit consequently to rarefaction taken place with the supersonic expansion. In the region of highest expansion, $z=8.5 \mathrm{~cm}$, it was possible to determine $n_{e}$ only over a limited radial extent. At 6 mbar, measurements are critically limited to the radial range $24<r<28 \mathrm{~mm}$. The lack of measurements induces some undesirable numerical artifacts when rebuilding the interpolated distribution with incomplete radial information. For instance, in the case $P_{c}=12 \mathrm{mbar}$, it is clear that the electron density increases at the jet edges at the proximity of the first compression, but its rise at centerline does not appear plausible, otherwise it would have been measured. That stands too for the 6 mbar case, preventing from the reliable characterization of the structure of the rarefaction cell after the Mach disk.

The resulting interpolated distributions for $T_{e x}$ are given in Fig. (21). The black lines indicate the actual radial extent of $T_{e x}$ measurements which ranges from $\sim 5000$ to $\sim 14000 \mathrm{~K}$. Temperature determination with Eq. (10) is not tractable in regions of lowest emission where, as consequence of the rarefaction local emission nearly fully vanished. Similarly to $n_{e}$ distribution situations, interpolation of measured $T_{e x}$ profiles suffers from the lack of data in the central part of the jet, generating unreliable distribution in regions of weakest emission. In spite of such inconvenient artifacts, the rebuilt distributions remain fully reliable in regions of actual measurements. The resulting distributions stand as a valuable data to serve as a test case to assess compressible plasma flow computations.

\section{CONCLUSION AND PERSPECTIVES}

The reported investigations address the characterization of four distinct non-equilibrium plasma kinds to produce new data valuable to assess the performances of nonequilibrium plasma flow descriptions.

High enthalpy shock tube experiments are well suited to procure critical data to assess the sophisticated descriptions developed to predict kinetic processes taking place behind the shock. The measurements of the emission of air postshock plasma generated with the high enthalpy shock tube at Institute for Problems in Mechanics (Russian Federation) have been documented and shortly analyzed. The radiative signatures measured in absolute radiance in the UV spectral range for an extended range of shock velocity stands as the most relevant data to validate post-shock non-equilibrium plasma flow computations determined on the basis of up-todate CR models.

The experimental electrical characterization of a shock wave, generated by the acoustic shock tube at University of Bari (Italy), propagating at nearly Mach 1 through a weakly ionized medium generated with a DC discharge, has been performed using laser deflection technique.

Detailed Particle in Cell-Monte Carlo (PIC-MC) investigations have been conducted to evaluate the effect of the resulting self-consistent electric force on the shock wave dynamic.

The characterization in terms of electron density $n_{e}$ and excitation temperature $T_{e x}$ of the subsonic nitrogen plasma jet produced by DC-Plasmatron facility at Poznan University of Technology (Poland) has demonstrated that the flowing plasma is far from the Saha equilibrium. The observed departure to equilibrium thermodynamic state indicates that the numerical rebuilding of the nitrogen subsonic flow has to be performed with an incompressible fluid model consistently coupled with a critically selected non-equilibrium chemistry description.

Moderately and highly under-expanded supersonic air plasma jets, produced by VKI-Plasmatron facility at von Karman institute (Belgium), have been investigated by uncovering the general features of the local emission, electron density $n_{e}$ and excitation temperature $T_{e x}$ distributions. The spatial characterization of the first expansion, the Barrel shock regions, the Mach disk and its post-shock area yielded relevant information to enhance the assessment of compressible plasma flow predictions. For instance, for the two considered conditions, the Mach disk location estimated with local emission distribution was found in good agreement with the empirical prediction. Further plasma jet 

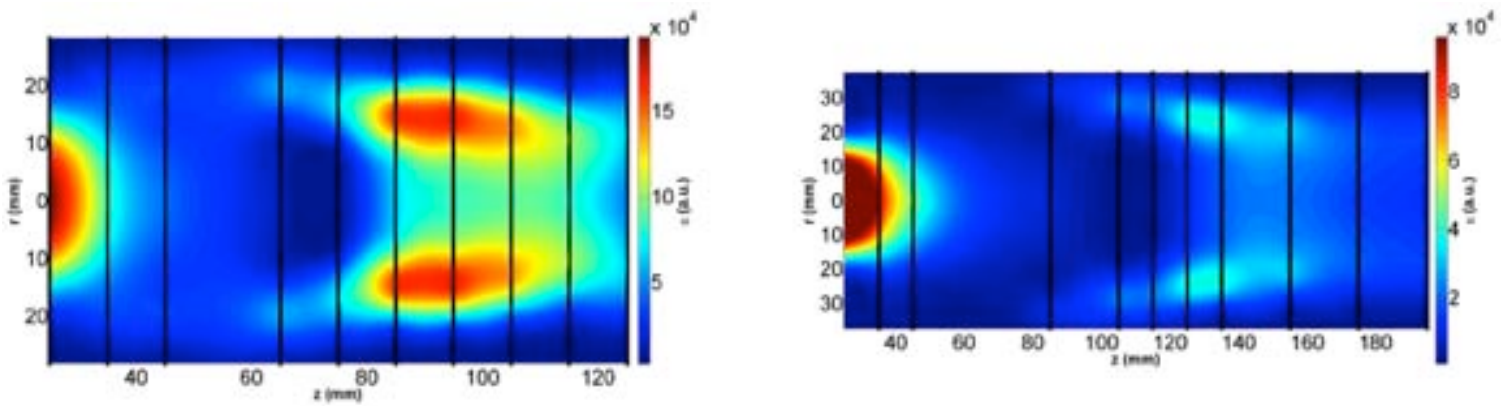

Fig. (19). $\varepsilon$ distributions with $P_{c}=12 \mathrm{mbar}$ (on left) and $P_{c}=6 \mathrm{mbar}$ (on right).
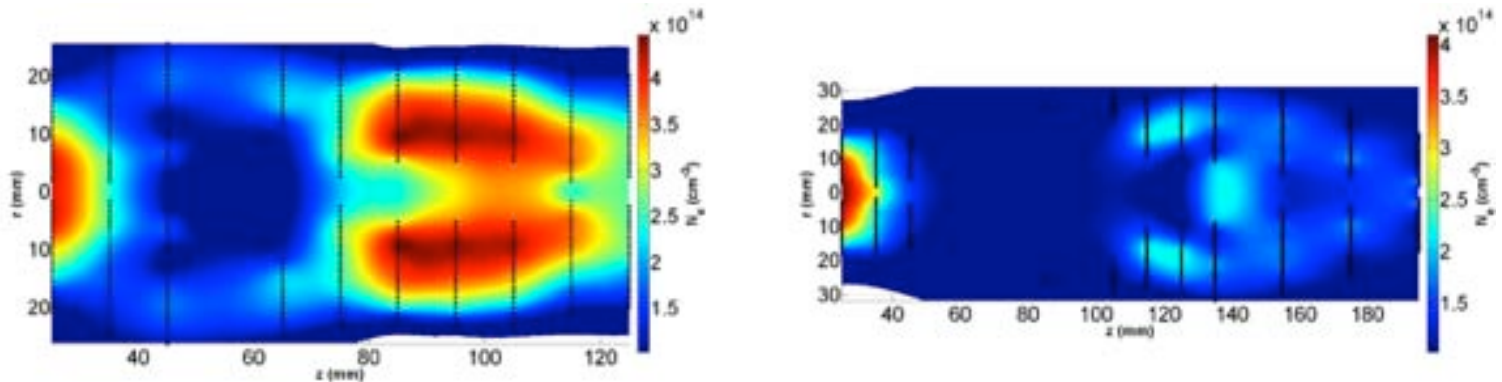

Fig. (20). $n_{e}$ distributions with $P_{c}=12 \mathrm{mbar}$ (on left) and $P_{c}=6 \mathrm{mbar}$ (on right).
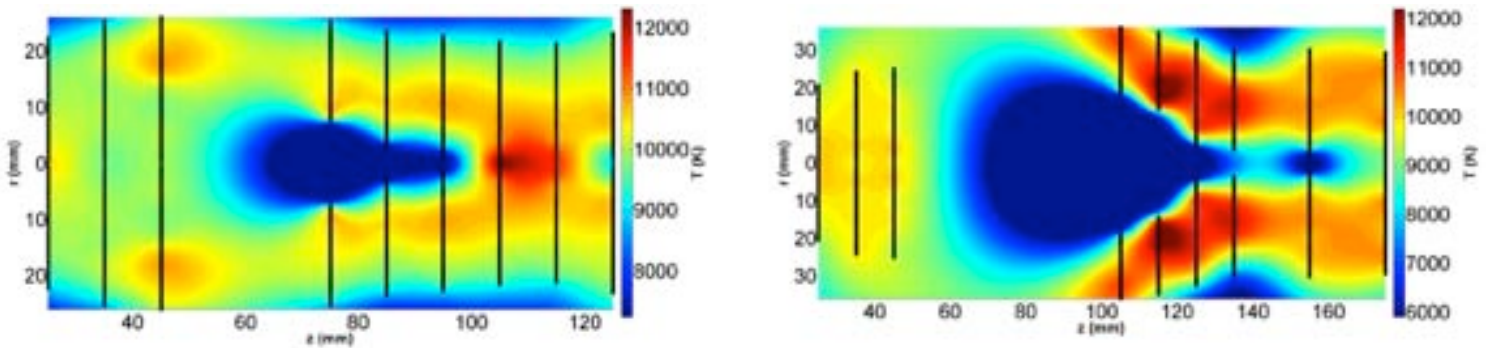

Fig. (21). $T_{e x}$ distributions with $P_{c}=12 \mathrm{mbar}$ (on left) and $P_{c}=6 \mathrm{mbar}$ (on right).

computations have to be carried out with $2 \mathrm{D}$ axisymmteric compressible fluid description coupled with a relevant nonequilibrium description to tackle regions where the imbalance kinetic processes are driven by recombining processes (e.g. in the expansion) or dominated by ionizing collisions (e.g. in the post-shock region).

\section{CONFLICT OF INTEREST}

The authors confirm that this article content has no conflict of interest.

\section{ACKNOWLEDGEMENTS}

The research leading to these results has received funding from the European Community Seventh Framework Programme (FP7/2007-2013) under Grant Agreement No. 242311.

\section{REFERENCES}

[1] Cauchon DL, McKee CW, Cornette ES. Radiative heating results from the fire II flight experiment at a reentry velocity of 11.4 kilometers per second. Technical report No. NASA TM X-1402. Langley Research Center 1967.

[2] Keck JC, Camm JC, Kivel B, Wentink Jr T. Radiation from hot air part II. Shock tube study of absolute intensities. Ann Phys 1959; 7(1): 1-38.

Available from: http:/www.sciencedirect.com/science/article/pii/0003491659900454
[3] Thomas GM, Menard WA. Experimental measurements of equilibrium and non-equilibrium radiation from planetary atmospheres. AIAA J. 1966; 4(2): 227-37

Available from: http://arc.aiaa.org/doi/abs/10.2514/3.3423

[4] Grinstead JH, Olejniczak J, Wilder MC, Bogdanoff MW, Allen GA, Lilliar R. Shock-heated air radiation measurements at lunar return conditions: Phase I EAST test report. Report No. NASA EG-CAP-07-142. NASA 2007.

[5] Kozlov PV, Romanenko YuV, Shatalov OP. Radiation intensity measurement in simulated Martian atmospheres on the double diaphragm shock tube. Proceedings of 4th International Workshop on Radiation of High Temperature Gases in Atmospheric Entry; ESA SP-689. Lausanne, Swiss, 2010.

[6] Takayanagi H, Fujita K. Absolute radiation measurements behind strong shock wave in carbondioxide flow for mars aerocaprure missions. AIAA paper 2012-2744.

Available from: http://arc.aiaa.org/doi/abs/10.2514/6.2012-2744

[7] Cruden BA, Prabhu D, Martinez R, Le H, Bose D, Grinstead JH. Absolute radiation measurement in venus and mars entry conditions. AIAA paper 2010-4508.

Available from: http://arc.aiaa. org/doi/abs/10.2514/6.2010-4508

[8] Grinstead JH, Wilder MC, Wright MJ, et al. Shock radiation measurements for mars aerocapture radiative heating analysis. AIAA paper 2008-1272.

Available from: http://arc.aiaa.org/doi/abs/10.2514/6.2008-1272

[9] Brandis AM, Morgan RG, McIntyre TJ, Jacobs PA. Nonequilibrium radiation intensity measurements in simulated titan atmospheres. J Thermophys Heat Transf 2010; 24: 291-300. Available from: http://arc.aiaa.org/doi/abs/10.2514/1.44482 
[10] Rond C, Boubert P, Félio JM, Chikhaoui A. Radiation measurements in shock tube for titan mixtures. J Thermophys Heat Transf 2007; 21(3): 638-46.

Available from: http://arc.aiaa.org/doi/abs/10.2514/1.28422

[11] Park C. Review of chemical-kinetic problems of future NASA missions, I: Earth entries. J Thermophys Heat Transf 1993; 7(13): 385-98. Available from: http://arc.aiaa.org/doi/abs/10.2514/3.431

[12] Park C, Howe JH, Jaffe RL, GV Candler. Review of chemicalkinetic problems of future NASA missions, II: mars entries. J Thermophys Heat Transf 1994; 8(1): 9-23.

Available from: http://arc.ai aa.org/doi/abs/10.2514/3.496

[13] Boubert P, Chaix A, Chikhaoui A, Robin L, Vervisch P. Aerodynamic calibration of TCM2 facility and study of a bow shock layer by emission and laser spectroscopy. Shock Waves 2002; 11(5): 341- 51. Available from: http://link.springer.com/article/10.1007

[14] Boubert P, Rond C. Non-equilibrium radiation in shocked martian mixtures. J Thermophys Heat Transf 2010; 24: 40-9. Available from: http://arc.aiaa.org/doi/abs/10.2514/1.45385

[15] Panesi M, Magin TE, Bourdon A, Bultel A, Chazot O, Babou Y. Collisional radiative modeling in flow simulation. VKI Lecture Series 2008; RTO-EN-AVT-162 - ISBN 978-92-837-0091-3.

[16] Brandis AM, Johnston CO, Cruden BA, et al. Validation of CO 4th positive radiation for mars entry. J Quant Spectrosc Radiat Transf 2013; 121: 91-104.

Available from: http:/www.sciencedirect.com/science/article/pii/S0022407313000630

[17] Cicala G, Bruno D, Capitelli M, Longo S, Rainò AC. Study of shock waves interacting with $\mathrm{Ar}$ and $\mathrm{N}_{2}$ low pressure de discharges. Eur Phys J D. 2010; 57(3): 375-85.

Available from: http://link.springer.com/article/10.1140

[18] Ionikh YZ, Chernysheva NV, Meschchanov AV, Yalin AP, Miles RB. Direct evidence for thermal mechanism of plasma influence on shock wave propagation. Phys Lett A 1999; 259(5): 387-92. Available from: http:/www.sciencedirect.com/science/article/pii/S0375960199004521

[19] Macheret SO, Ionikh YZ, Chernysheva NV, Yalin AP, Martinelli L, Miles RB. Shock wave propagation and dispersion in glow discharge plasmas. Phys Fluids 2001; 13: 2693-705. Available from: http://dx.doi.org/10.1063/1.1388204

[20] Aihtal SM, Subramaniam VV. On the characteristics of a spark generated shock wave. Phys Fluids 2000; 12: 924-35. Available from: http://dx.doi.org/10.1063/1.870345

[21] White AR, Subramaniam VV. Shock propagation through a lowpressure glow discharge in argon. J Thermophys Heat Transf 2011; 15: 491-6. Available from: http://arc.aiaa.org/doi/abs/10.2514/2.6638

[22] Klimov AI, Koblov AN, Mishin GI, Serov YuL, Yavor IP. Shock wave propagation in a glow discharge Sov Tech Phys Lett 1982; 8: $192-4$

[23] Bletzinger P, Ganguly BN. Local acoustic shock velocity and shock structure recovery measurements in glow discharges. Phys Lett A 1999; 258(4-6): 342-8.

Available from: $h$ ttp:/www.science direct.com/science/article/pii/S0375960199003862

[24] Bletzinger P, Ganguly BN, Garscadden A. Electric field and plasma emission responses in a low pressure positive column discharge exposed to a low Mach number shock wave. Phys Plasmas 2000; 7(10): 4341-7.

Available from: http://dx.doi.org/10.1063/1.1287218

[25] Fischer WPP, Gelhan A, Esser B, Faerber J, Reger N. Plasmawindtunnel tests on a new metallic TPS material, hot structures and thermal protection systems for space vehicles. Proceedings of the 4th European Workshop; 2002 Nov 26-29; Palermo, Italy. Ed. Wilson A. ESA SP-521. Paris: European Space Agency 2003; p. 199 .

[26] Caristia S, De Filippis D. SCIROCCO project a major plasma wind tunnel for the year 2000. Proceedings of the $1^{\text {st }}$ International Symposium on Atmospheric Reentry Vehicles and Systems; Arcachon, France, AAAF, 1999.
[27] Bottin B, Chazot O, Carbonaro M, Van Der Haagen V, Paris S Measurement techniques for high enthalpy and plasma flows 2000 , RTO-EN-8, AC/323(AVT)TP/23, 8 .

[28] Gordeev AN. Overview of characteristics and experiments in IPM plasmatrons. RTO AVT/VKI Special Course on Measurement Techniques for High Enthalpy Plasma Flows, RTO EN-1, von Karman Institute for Fluid Dynamics. Belgium: Rhode-SaintGenèse 1999.

[29] Lequang D, Babou Y, André P. Experimental assessment of local thermodynamic equilibrium in VKI Plasmatron air plasma jet. J Tech Phys 2009; 50(3): 151-62.

[30] Laux CO, Pierrot L, Gessman RJ. State-to-state modeling of a recombining nitrogen plasma experiment. Chem Phys 2012; 398(4): 46-55. Available from: http://www.sciencedirect.com/scie nce/article/pii/S0301010411004617

[31] Van de Sanden MCM, de Regt JM, Schram DC. Recombination of argon in an expanding plasma jet. Phys Rev E 1993; 47(4): 279297.

Available from: http://link.aps.org/doi/10.1103/PhysRevE.47.2792

[32] Sember V, Gravelle DV, Boulos MI. Spectroscopic study of a supersonic plasma jet generated by an ICP torch with a convergentdivergent nozzle. J Phys D 2002; 35(12): 1350-61. Available from: http://iopscience.iop.org/0022-3727/35/12/311

[33] Spanier J, Gelbard EM. Monte Carlo principles and neutron transport problems. Addison Wesley; 1969, reprinted Dover 2008.

[34] Dupree SA, Fraley SK. Monte Carlo primer: practical approach to radiation transport. Kluwer 2002.

[35] Hockney RW, Eastwood WR. Computer simulation using particles. Adam Hilger 1991.

[36] Drawin HW, Emard F. Optical escape factors for bound-bound and free-bound radiation from plasmas. I. Constant source function. Beiträge aus der Plasmaphys 1973 ; 13(3): 143-68. Available from: http://onlinelibrary.wiley.com/doi/10.1002/ctpp.19730130304/abstract

[37] Richter J. Plasma diagnostics. North Holland, Amsterdam: LochteHoltgreven 1968

[38] Available from: http//www.astronomy.ohio-state.edu/nahar/nahar_radiativeatomicdata/index.html

[39] Schlüter D. Gaunt factors for free-free transitions in the fields of positive ions of carbon, nitrogen and oxygen calculated in Scaled Thomas-Fermi approximation. Z Phys D - Atoms, Molecules and Clusters. 1989; 11(4): 273-6.

Available from: http://link.springer.com/article/10.1007

[40] Cabannes F, Chapelle JC. Reactions under plasma conditions. New York: M.Venugopalan, Wiley; 1971.

[41] Wilbers ATM, Kroesen GMW, Timmermans CJ, Schramm DC. The continuum emission of an arc plasma. J Quant Spectrosc Radiat Transf 1991; 45(1): 1-10.

Available from: $\mathrm{http}: /$ www.sciencedirect.com/science/article/pii/0022407391900763

[42] Capitelli M, Devoto RS. Transport coefficients of high-temperature nitrogen. Phys Fluids 1973; 16(11): 1841-75. Available from: http://dx.doi.org/10.1063/1.1694222

[43] Lino da Silva M. Simulation des propriétés radiatives du plasma entourant un véhicule traversant une atmosphère planétaire à vitesse hypersonique - application à la planète Mars. Ph.D. diss., Université d'Orléans 2004

[44] McWhirter RWP. Plasma diagnostics techniques. New York: R.H. Huddelstone and S.L. Leonard Academic Press 1965.

[45] Available from: http://physics.nist.gov/asd2i

[46] Gigosos MA, Cardeñoso V. New plasma diagnosis tables of hydrogen Stark broadening including ions dynamics. J Phys B 1996; 29(20): 4795-838.

Available from: http://iopscience.iop.org/0953-4075/29/20/029

[47] Ashkenas H, Sherman FS. The structure and utilization of supersonic free jets in low density wind tunnels. Proc 4th Int Symp on Rarefied Gas Dynamics 1996; 2: 84-105. 